TRANSACTIONS OF THE

AMERICAN MATHEMATICAL SOCIETY

Volume 363, Number 4, April 2011, Pages 1947-1980

S 0002-9947(2010)05303-6

Article electronically published on November 15, 2010

\title{
ANALYSIS OF SPECTRAL METHODS FOR THE HOMOGENEOUS BOLTZMANN EQUATION
}

\author{
FRANCIS FILBET AND CLÉMENT MOUHOT
}

\begin{abstract}
The development of accurate and fast algorithms for the Boltzmann collision integral and their analysis represent a challenging problem in scientific computing and numerical analysis. Recently, several works were devoted to the derivation of spectrally accurate schemes for the Boltzmann equation, but very few of them were concerned with the stability analysis of the method. In particular there was no result of stability except when the method was modified in order to enforce the positivity preservation, which destroys the spectral accuracy. In this paper we propose a new method to study the stability of homogeneous Boltzmann equations perturbed by smoothed balanced operators which do not preserve positivity of the distribution. This method takes advantage of the "spreading" property of the collision, together with estimates on regularity and entropy production. As an application we prove stability and convergence of spectral methods for the Boltzmann equation, when the discretization parameter is large enough (with explicit bound).
\end{abstract}

\section{Contents}

1. Introduction

2. The Boltzmann equation

3. Formulation of a general stability result 1951

3.1. General framework 1952

3.2. The truncation associated with classical spectral methods 1954

3.3. The truncation associated with fast spectral methods 1954

3.4. A common abstract formulation for the stability of spectral methods

4. Proof of stability on an arbitrary bounded time interval 1957

4.1. Preliminary results 1957

4.2. Existence and regularity on a bounded time interval 1964

5. Asymptotic behavior and global in time stability 1966

5.1. Regularity study of the periodized Boltzmann equation 1966

5.2. Entropy - entropy production inequalities $\quad 1970$

Received by the editors December 2, 2008.

2000 Mathematics Subject Classification. Primary 82C40, 65M70, 76P05.

Key words and phrases. Boltzmann equation, spectral methods, numerical stability, asymptotic stability, Fourier-Galerkin method.

The first author would like to express his gratitude to the ANR JCJC-0136 MNEC (Méthode Numérique pour les Equations Cinétiques) and to the ERC StG \#239983 (NuSiKiMo) for funding.

The second author would like to thank Cambridge University who provided repeated hospitality in 2009 thanks to the Award No. KUK-I1-007-43, funded by the King Abdullah University of Science and Technology (KAUST).

(C)2010 American Mathematical Society Reverts to public domain 28 years from publication 1947 
5.3. Proof of the global in time stability

6. Application: Stability and convergence of spectral methods 1976

\begin{tabular}{ll|l|}
6.1. & Proof of Theorem 6.1 & 1978 \\
\hline
\end{tabular}

References $\quad 1979$

\section{INTRODUCTION}

This work is the sequel of several papers devoted to the approximation of the Boltzmann equation using fast spectral methods [27, 11, 21, 14]. The present paper is devoted to the stability and convergence analysis of general spectral algorithms.

In a microscopic description of rarefied gas, the particles move by a constant velocity until they undergo binary collisions. In statistical physics, the properties of the gas are described by a density function in phase space, $f(t, x, v)$, called the distribution function, which gives the fraction of particles per unit volume in phase space at time $t$. The distribution function satisfies the Boltzmann equation, a nonlinear integro-differential equation, which describes the combined effect of the free flow and binary collisions between the particles.

The main difficulty in the approximation of the Boltzmann equation is due to the multi-dimensional structure of the collisional integral, since the integration runs on a highly-dimensional unflat manifold. In addition, the numerical integration requires great care, since the collision integral is at the basis of the macroscopic properties of the equation. Further difficulties are represented by the presence of stiffness, such as the case of a small mean free path or the case of large velocities [10].

For such reasons realistic numerical simulations are based on Monte-Carlo techniques. The most famous examples are the Direct Simulation Monte-Carlo (DSMC) methods by Bird 11 and by Nanbu 22. These methods guarantee efficiency and preservation of the main physical properties. However, avoiding statistical fluctuations in the results becomes extremely expensive in the presence of non-stationary flows or close to continuum regimes.

Among deterministic approximations, Discrete Velocity Models (DVM) are based on a Cartesian grid in velocity and on a discrete collision mechanism on the points of the grid that preserves the main physical properties. Unfortunately DVM are not competitive with Monte-Carlo methods in terms of computational cost, and their accuracy seems to be less than first order [23, 24, 25, 9.

Another class of numerical methods, based on the use of spectral techniques in the velocity space, has been developed. The methods were first derived in [26, inspired from spectral methods in fluid mechanics [6] and by previous works on the use of Fourier transform techniques for the Boltzmann equation [2]. They are based on approximating in the velocity space the distribution function by a periodic function, and on its representation by Fourier series. The spectral method has been further developed in [27, 29] where evolution equations for the Fourier modes were explicitly derived and spectral accuracy of the method was proven. Strictly speaking, these methods are not conservative, since they only preserve mass, whereas momentum and energy are approximated with spectral accuracy. Moreover, the spectral method has also been applied to non-homogeneous situations [11, to the Landau equation [30, 10, 28, where fast algorithms can be readily derived, and to the case of granular gases [13. Independently A. Bobylev and S. Rjasanow [3, 4] 
have also constructed fast algorithms based on a Fourier transform approximation of the distribution function, but the method is not spectrally accurate (only second order).

In 21] a fast spectral method was proposed for a class of particle interactions including pseudo-Maxwell molecules in dimension 2 and, most importantly, hard spheres in dimension 3, on the basis of the previous spectral method together with a suitable semi-discretization of the collision operator. This method permits us to reduce the computational cost from $O\left(n^{2}\right)$ to $O\left(n \log _{2} n\right)$ without losing the spectral accuracy, thus making the method competitive with Monte-Carlo.

However, an important drawback of the spectral methods up to now has been the lack of proof of stability. Indeed, as compared to discrete velocity methods, the difficulties are somehow opposite: consistency results are easily obtained, whereas the lack of positivity preservation of the scheme is a major issue when one studies its stability properties. The only paper concerned with the issue of stability for spectral methods applied to the Boltzmann collision operator is [29], but in the latter the author introduces some "filters" on the Fourier modes in order to restore the positivity-preservation of the scheme, which breaks the spectral accuracy.

In this paper we give the first stability result for the spectral methods applied to the Boltzmann collision operator. Moreover, we propose a method which is likely to have other utilizations in collisional kinetic theory:

- we write the Galerkin approximation on the first $N$ Fourier modes of the evolution equation as a smooth balanced perturbation of the original equation, in the sense of a perturbation by some small and mass-preservation (although not positivity-preserving) error term;

- we prove existence and uniqueness of smooth solutions for small times, conditionally to a bound on the $L^{1}$ norm;

- we use the mixing structure [31, 19] of the collision process to show the appearance of positivity after a small time (depending on the size of the box of truncation and the approximation parameter $N$ );

- we use the mass conservation to deduce uniform bounds on the $L^{1}$ norm, and therefore regularity bounds growing at most exponentially in time;

- we perform a detailed analysis of the unperturbed truncated problem, showing uniform in time regularity and asymptotic convergence to equilibrium;

- finally we use the fact that the equilibrium is unchanged by the smooth balanced perturbation and that it is non-linearly stable for the perturbed periodized Boltzmann equation, in order to prove global in time stability and convergence to equilibrium for the perturbed Boltzmann equation (we connect the previous point for initial times, together with the stability of equilibrium for asymptotic times).

Hence our paper introduces a general method on how to exploit fine mixing properties of the collision process in the study of stability of a particular class of perturbed Boltzmann equation, with the application in mind to the stability of spectral methods.

The outline of this paper is as follows. The Boltzmann equation and its basic features are presented in Section 2. In Section 3 we explain the truncation and periodization associated with spectral methods and fast spectral methods. We also formulate the problem of stability of these methods in the general framework of the stability properties of the Boltzmann equation with respect to a smooth balanced 
perturbation. Section 4 is devoted to the proof of the main stability result in the general framework. Section [5 is devoted to the study of the asymptotic behavior of the truncated problem on the basis of the entropy production theory. Finally, in Section [6 we apply the latter result to the spectral method and establish some stability and convergence results of the numerical solution.

\section{The Boltzmann EQUATion}

The Boltzmann equation describes the behavior of a dilute gas of particles when the only interactions taken into account are binary elastic collisions. It reads for $x \in \Omega, v \in \mathbb{R}^{d}$, where $\Omega \in \mathbb{R}^{d}$ is the spatial domain $(d \geq 2)$ :

$$
\frac{\partial f}{\partial t}+v \cdot \nabla_{x} f=Q(f, f)
$$

where $f:=f(t, x, v)$ is the time-dependent particle distribution function in the phase space. The Boltzmann collision operator $Q$ is a quadratic operator local in $(t, x)$. The time $t$ and position $x$ act only as parameters in $Q$, and therefore will be omitted in its description:

$$
Q(f, f)(v)=\int_{v_{\star} \in \mathbb{R}^{d}} \int_{\sigma \in \mathbb{S}^{d-1}} B\left(\left|v-v_{\star}\right|, \cos \theta\right)\left(f_{\star}^{\prime} f^{\prime}-f_{\star} f\right) d \sigma d v_{\star} .
$$

We used the shorthand $f=f(v), f_{\star}=f\left(v_{\star}\right), f^{\prime}=f\left(v^{\prime}\right), f_{\star}^{\prime}=f\left(v_{\star}^{\prime}\right)$. The velocities of the colliding pairs $\left(v, v_{\star}\right)$ and $\left(v^{\prime}, v_{\star}^{\prime}\right)$ are related by

$$
\left\{\begin{array}{l}
v^{\prime}=v-\frac{1}{2}\left(\left(v-v_{\star}\right)-\left|v-v_{\star}\right| \sigma\right), \\
v_{\star}^{\prime}=v-\frac{1}{2}\left(\left(v-v_{\star}\right)+\left|v-v_{\star}\right| \sigma\right),
\end{array}\right.
$$

with $\sigma \in \mathbb{S}^{d-1}$. The collision kernel $B$ is a non-negative function which by physical arguments of invariance only depends on $\left|v-v_{\star}\right|$ and $\cos \theta=u \cdot \sigma$, where $u=$ $\left(v-v_{\star}\right) /\left|v-v_{\star}\right|$ is the normalized relative velocity.

In this work we are concerned with short-range interaction models. More precisely we assume that $B$ is locally integrable. Here are the hypothesis on the collision kernel:

$$
B(|u|, \cos \theta)=\Phi(|u|) b(\cos \theta),
$$

with

$$
\Phi(z)=z^{\gamma}, \quad z \in \mathbb{R}_{+}, \quad \text { for some } \gamma \in(0,+\infty)
$$

and $b$ smooth such that

$$
\int_{0}^{\pi} b(\cos \theta) \sin ^{d-2} \theta d \theta<+\infty .
$$

These assumptions are satisfied for the so-called hard spheres model $B(u)=|u|$, and it is known as Grad's angular cutoff assumption when it is (artificially) extended to interactions derived from a power-law potential. As an important benchmark model for the numerical simulation, we therefore introduce the so-called variable hard spheres model (VHS), which writes

$$
B(|u|, \cos \theta)=C_{\gamma}|u|^{\gamma}
$$

for some $\gamma \in(0,1]$ and a constant $C_{\gamma}>0$. 
For this class of model, one can split the collision operator as

$$
Q(f, f)=Q^{+}(f, f)-L(f) f
$$

with

$$
Q^{+}(f, f)=\int_{\mathbb{R}^{d}} \int_{\mathbb{S}^{d-1}} B\left(\left|v-v_{\star}\right|, \cos \theta\right) f^{\prime} f_{\star}^{\prime} d \sigma d v_{\star}
$$

and

$$
L(f)=\int_{\mathbb{R}^{d}} \int_{\mathbb{S}^{d-1}} B\left(\left|v-v_{\star}\right|, \cos \theta\right) f_{\star} d \sigma d v_{\star} .
$$

Boltzmann's collision operator has the fundamental properties of conserving mass, momentum and energy: at the formal level

$$
\int_{\mathbb{R}^{d}} Q(f, f) \phi(v) d v=0, \quad \phi(v)=1, v,|v|^{2},
$$

and it satisfies well-known Boltzmann's $H$ theorem

$$
-\frac{d}{d t} \int_{\mathbb{R}^{d}} f \log f d v=-\int_{\mathbb{R}^{d}} Q(f, f) \log (f) d v \geq 0 .
$$

The functional $-\int f \log f$ is the entropy of the solution. Boltzmann's $H$ theorem implies that any equilibrium distribution function, i.e., any function which is a maximum of the entropy, has the form of a locally Maxwellian distribution

$$
M(\rho, u, T)(v)=\frac{\rho}{(2 \pi T)^{d / 2}} \exp \left(-\frac{|u-v|^{2}}{2 T}\right),
$$

where $\rho, u, T$ are the density, macroscopic velocity and temperature of the gas, defined by

$$
\rho=\int_{v \in \mathbb{R}^{d}} f(v) d v, \quad u=\frac{1}{\rho} \int_{v \in \mathbb{R}^{d}} v f(v) d v, \quad T=\frac{1}{d \rho} \int_{v \in \mathbb{R}^{d}}|u-v|^{2} f(v) d v .
$$

For further details on the physical background and derivation of the Boltzmann equation we refer to Cercignani, Illner, Pulvirenti [8] and Villani [33.

\section{Formulation of a General Stability Result}

In this section we recall the basic principles leading to the periodized truncations of the Boltzmann collision operator arising in spectral methods. Then we present the main result of this paper: the stability of the spatially homogeneous Boltzmann equation with respect to a smooth balanced perturbation, preserving mass and smoothness but not non-negativity of the solution. This stability means that we are able to construct global solutions and estimate the error between perturbed and unperturbed solutions.

Any deterministic numerical method requires us to work on a bounded velocity space. This therefore supposes a non-physical truncation (associated with limit conditions) of this velocity space, which we shall discuss below. 
3.1. General framework. We consider the spatially homogeneous Boltzmann equation written in the following general form:

$$
\frac{\partial f}{\partial t}=Q(f, f)
$$

where $Q(f, f)$ is given by

$$
Q(f, f)=\int_{\mathcal{C}} \mathcal{B}(y, z)\left[f^{\prime} f_{\star}^{\prime}-f_{\star} f\right] d y d z, \quad v \in \mathbb{R}^{d},
$$

with

$$
v^{\prime}=v+\Theta^{\prime}(y, z), \quad v_{\star}^{\prime}=v+\Theta_{\star}^{\prime}(y, z), \quad v_{\star}=v+\Theta_{\star}(y, z) .
$$

In the equations above, $\mathcal{C}$ is some given (unbounded) domain for $y, z$, and $\Theta, \Theta^{\prime}$, $\Theta_{\star}^{\prime}$ are suitable functions, to be defined later. This general framework emphasizes the translation invariance property of the collision operator, which is crucial for the spectral methods. We will be more precise in the following paragraphs for some changes of variables, allowing us to reduce the classical operator (2.1) to the form (3.2).

A problem associated with deterministic methods which use a fixed discretization in the velocity domain is that the velocity space is approximated by a finite region. Physically the domain for the velocity is $\mathbb{R}^{d}$, and the property of having compact support is not preserved by the collision operator. In general the collision process indeed spreads the support by a factor $\sqrt{2}$ in the elastic case (see [31, 19] and also [18 for similar properties in the inelastic case). As a consequence, for the continuous equation in time, the function $f$ is immediately positive in the whole domain $\mathbb{R}^{d}$. Thus, at the numerical level, some non-physical condition has to be imposed to keep the support uniformly bounded. In order to do this there are two main strategies:

- One can remove the physical binary collisions that will lead outside the bounded velocity domains. This means a possible increase in the number of local invariants, i.e., the functions $\varphi$ such that

$$
\left(\varphi_{\star}^{\prime}+\varphi^{\prime}-\varphi_{\star}-\varphi\right)
$$

is zero everywhere on the domain. If this is done properly (i.e., without removing too many collisions), the scheme remains conservative and without spurious invariants. However, this truncation breaks down the convolutionlike structure of the collision operator, which requires the translation invariance in velocity. Indeed the modified collision kernel depends on $v$ through the boundary conditions. This truncation is the starting point of most schemes based on Discrete Velocity Models.

- One can add some non-physical binary collisions by periodizing the function and the collision operator. This implies the loss of some local invariants (some non-physical collisions are added). Thus the scheme is not conservative anymore, although it still preserves the mass if the periodization is done carefully. However, in this way the structural properties of the collision operator are maintained, and thus they can be exploited to derive fast algorithms. This periodization is the basis of spectral methods.

Therefore, we consider the space homogeneous Boltzmann equation in a bounded domain in velocity $\mathcal{D}_{L}=[-L, L]^{d}$ with $0<L<\infty$. We truncate the integration in $y$ and $z$ in (3.2) since periodization would yield an infinite result if not: we set $y$ and 
$z$ to belong to some truncated domain $\mathcal{C}_{R} \subset \mathcal{C}$ (the parameter $R$ refers to its size and will be defined later). For a compactly supported function with support included in $\mathcal{B}_{S}$, the ball centered at 0 with radius $S>0$, one has to prescribe suitable relations (depending on the precise change of variable and truncation chosen) between $S$, $R$ and $L$ in order to retain all possible collisions and at the same time prevent intersections of the regions where $f$ is different from zero (this is the so-called dealiasing condition). Then the truncated collision operator reads

$$
Q^{R}(f, f)=\int_{\mathcal{C}_{R}} \mathcal{B}(y, z)\left(f_{\star}^{\prime} f^{\prime}-f_{\star} f\right) d y d z
$$

for $v \in \mathcal{D}_{L}$ (the expression for $v \in \mathbb{R}^{d}$ is deduced by periodization). By making some changes of variable on $v$, one can easily prove for the two choices of variables $y, z$ of the next subsections that for any function $\varphi$ periodic on $\mathcal{D}_{L}$ the following weak form is satisfied:

$$
\int_{\mathcal{D}_{L}} Q^{R}(f, f) \varphi(v) d v=\frac{1}{4} \int_{\mathcal{D}_{L}} \int_{\mathcal{C}_{R}} \mathcal{B}(y, z) f_{\star} f\left(\varphi_{\star}^{\prime}+\varphi^{\prime}-\varphi_{\star}-\varphi\right) d y d z d v .
$$

Now, we use the representation $Q^{R}$ to derive spectral methods. Hereafter, we use just one index to denote the $d$-dimensional sums with respect to the vector $k=\left(k_{1}, \ldots, k_{d}\right) \in \mathbb{Z}^{d}$. Hence we set

$$
\sum_{k=-N}^{N}:=\sum_{k_{1}, \ldots, k_{d}=-N}^{N} .
$$

The approximate function $f_{N}$ is represented as the truncated Fourier series

$$
f_{N}(v)=\sum_{k=-N}^{N} \hat{f}_{k} e^{i \frac{\pi}{L} k \cdot v}
$$

with

$$
\hat{f}_{k}=\frac{1}{(2 L)^{d}} \int_{\mathcal{D}_{L}} f(v) e^{-i \frac{\pi}{L} k \cdot v} d v .
$$

In a Fourier-Galerkin method the fundamental unknowns are the coefficients $\hat{f}_{k}(t)$, $k=-N, \ldots, N$. We obtain a set of ODEs for the coefficients $\hat{f}_{k}$ by requiring that the residual of (3.3) be orthogonal to all trigonometric polynomials of degree less than $N$. Hence for $k=-N, \ldots, N$,

$$
\int_{\mathcal{D}_{L}}\left(\frac{\partial f_{N}}{\partial t}-Q^{R}\left(f_{N}, f_{N}\right)\right) e^{-i \frac{\pi}{L} k \cdot v} d v=0 .
$$

By substituting expression (3.5) in (3.4) we get

$$
Q^{R}\left(f_{N}, f_{N}\right)=Q^{R,+}\left(f_{N}, f_{N}\right)-L^{R}\left(f_{N}\right) f_{N}
$$

with

$$
\begin{aligned}
L^{R}\left(f_{N}\right) f_{N} & =\sum_{l=-N}^{N} \sum_{m=-N}^{N} \beta(m, m) \hat{f}_{l} \hat{f}_{m} e^{i \frac{\pi}{L}(l+m) \cdot v}, \\
Q^{R,+}\left(f_{N}, f_{N}\right) & =\sum_{l=-N}^{N} \sum_{m=-N}^{N} \beta(l, m) \hat{f}_{l} \hat{f}_{m} e^{i \frac{\pi}{L}(l+m) \cdot v},
\end{aligned}
$$


where

$$
\beta(l, m)=\int_{\mathcal{C}_{R}} \mathcal{B}(y, z) e^{i \frac{\pi}{L}\left(l \cdot \Theta^{\prime}(y, z)+m \cdot \Theta_{\star}^{\prime}(y, z)\right)} d y d z .
$$

The spectral equation is the projection of the collision equation in $\mathbb{P}_{N}$, the $(2 N+1)^{d}$-dimensional vector space of trigonometric polynomials of degree at most $N$ in each direction, i.e.,

$$
\frac{\partial f_{N}}{\partial t}=\mathcal{P}_{N} Q^{R}\left(f_{N}, f_{N}\right)
$$

where $\mathcal{P}_{N}$ denotes the orthogonal projection on $\mathbb{P}_{N}$ in $L^{2}\left(\mathcal{D}_{L}\right)$.

3.2. The truncation associated with classical spectral methods. In the classical spectral method [27, a simple change of variables in (2.1) permits us to write

$$
Q(f, f)=\int_{\mathbb{R}^{d}} \int_{\mathbb{S}^{d-1}} \mathcal{B}^{\text {class }}(g, \omega)\left(f\left(v^{\prime}\right) f\left(v_{\star}^{\prime}\right)-f(v) f\left(v_{\star}\right)\right) d \omega d g,
$$

with $g=v-v_{\star} \in \mathbb{R}^{d}, \omega \in \mathbb{S}^{d-1}$, and

$$
\left\{\begin{array}{l}
v^{\prime}=v-\frac{1}{2}(g-|g| \omega), \\
v_{\star}^{\prime}=v-\frac{1}{2}(g+|g| \omega), \\
v_{\star}=v+g .
\end{array}\right.
$$

Then, we set $\mathcal{C}:=\mathbb{R}^{d} \times \mathbb{S}^{d-1}$ and

$$
\Theta^{\prime}(g, \omega):=-\frac{1}{2}(g-|g| \omega), \quad \Theta_{\star}^{\prime}(g, \omega):=-\frac{1}{2}(g+|g| \omega), \quad \Theta_{\star}(g, \omega):=g .
$$

Finally the collision kernel $\mathcal{B}^{\text {class }}$ is defined by

$$
\mathcal{B}^{\text {class }}(g, \omega)=2^{d-1}(1-(\hat{g} \cdot \omega))^{d / 2-1} B\left(|g|, 2(\hat{g} \cdot \omega)^{2}-1\right) .
$$

Thus, the Boltzmann operator (3.10) is now written in the form (3.2). We consider the bounded domain $\mathcal{D}_{L}=[-L, L]^{d}$ for the distribution $f$ and the bounded domain $\mathcal{C}_{R}=\mathcal{B}_{R} \times \mathbb{S}^{d-1}$ for some $R>0$. The truncated operator reads in this case

$$
Q^{R}(f, f)(v)=\int_{\mathcal{B}_{R} \times \mathbb{S}^{d-1}} \mathcal{B}^{\text {class }}(g, \omega)\left(f\left(v_{*}^{\prime}\right) f\left(v^{\prime}\right)-f\left(v_{*}\right) f(v)\right) d \omega d g .
$$

3.3. The truncation associated with fast spectral methods. Here we shall approximate the collision operator starting from a representation which conserves more symmetries of the collision operator when one truncates it in a bounded domain. This representation was used in [3, 15, to derive finite difference schemes, and it is close to the classical Carleman representation (cf. [7]). The basic identity we shall need is (for $u \in \mathbb{R}^{d}$ )

$$
\frac{1}{2} \int_{\mathbb{S}^{d-1}} F(|u| \sigma-u) d \sigma=\frac{1}{|u|^{d-2}} \int_{\mathbb{R}^{d}} \delta\left(2 y \cdot u+|y|^{2}\right) F(y) d y .
$$

Using (3.14) the collision operator (2.1) can be written as

$$
\begin{aligned}
Q(f, f)(v)=2^{d-1} \int_{x \in \mathbb{R}^{d}} \int_{y \in \mathbb{R}^{d}} & \mathcal{B}^{\text {fast }}(y, z) \delta(y \cdot z) \\
& (f(v+z) f(v+y)-f(v+y+z) f(v)) d y d z
\end{aligned}
$$


with

$$
\mathcal{B}^{\text {fast }}(y, z)=2^{d-1} B\left(|y+z|,-\frac{y \cdot(y+z)}{|y||y+z|}\right)|y+z|^{-(d-2)} .
$$

Thus, the collision operator is now written in the form (3.2) with $\mathcal{C}:=\mathbb{R}^{d} \times \mathbb{R}^{d}$,

$$
\mathcal{B}(y, z)=\mathcal{B}^{\mathrm{fast}}(y, z) \delta(y \cdot z),
$$

and

$$
v_{\star}^{\prime}=v+\Theta_{\star}^{\prime}(y, z), \quad v^{\prime}=v+\Theta^{\prime}(y, z), \quad v_{\star}=v+\Theta_{\star}(y, z)
$$

with

$$
\Theta_{\star}^{\prime}(y, z):=z, \quad \Theta^{\prime}(y, z):=y, \quad \Theta_{\star}(y, z):=y+z .
$$

Now we consider the bounded domain $\mathcal{D}_{L}=[-L, L]^{d},(0<L<\infty)$ for the distribution $f$ and the bounded domain $\mathcal{C}_{R}=\mathcal{B}_{R} \times \mathcal{B}_{R}$ for some $R>0$. The (truncated) operator now reads

$$
Q^{R}(f, f)(v)=\int_{\mathcal{C}_{R}} \mathcal{B}^{\text {fast }}(y, z) \delta(y \cdot z)(f(v+z) f(v+y)-f(v+y+z) f(v)) d y d z
$$

for $v \in \mathcal{D}_{L}$. This representation of the collision kernel yields better decoupling properties between the arguments of the operator and allows us to lower significantly the computation cost of the method by using the fast Fourier transform (see [21, 14]).

Let us make a crucial remark about the choice of $R$. When $f$ has support included in $\mathcal{B}_{S}, S>0$, it is usual (see [27, 21]) to search for the minimal period $L$ (in order to minimize the computational cost) which prevents interactions between different periods of $f$ during one collision process. From now on, we shall always assume that we can take $L$ and $R$ large enough such that, when needed, $R \geq \sqrt{2} L$. Hence all the torus is covered (at least once) in the integration of the variables $(g, \omega)$ or $(y, z)$.

\subsection{A common abstract formulation for the stability}

of spectral methods. From now on, $Q^{R}$ shall denote a periodized truncated collision operator as in (3.13) or (3.16). As we shall see, using this formulation, both classical and fast spectral methods fall into the following framework:

$$
\left\{\begin{array}{l}
\frac{\partial f}{\partial t}=Q^{R}(f, f)+P_{\varepsilon}(f), \quad v \in \mathcal{D}_{L}, \\
f(0, v)=f_{0, \varepsilon}(v), \quad v \in \mathcal{D}_{L},
\end{array}\right.
$$

where $P_{\varepsilon}$ is a "smooth balanced perturbation", which means that it satisfies the following (balanced law)

$$
\int_{\mathcal{D}_{L}} P_{\varepsilon}(f) d v=0
$$

and preserves the smoothness of the distribution function, i.e., there exist constants $C_{0}, C_{k}>0$ such that

$$
\left\{\begin{array}{l}
\left\|P_{\varepsilon}(f)\right\|_{L^{1}} \leq C_{0}\|f\|_{L^{1}}\|f\|_{L^{1}}, \\
\left\|P_{\varepsilon}(f)\right\|_{H_{\mathrm{per}}^{k}} \leq C_{k}\|f\|_{L^{1}}\|f\|_{H_{\mathrm{per}}^{k}}, \quad k \geq 0,
\end{array}\right.
$$

where $\|\cdot\|_{H_{\mathrm{per}}^{k}}$ is the usual norm of the Sobolev space of periodic functions $H_{\mathrm{per}}^{k}\left(\mathcal{D}_{L}\right)$. 
Moreover, the perturbation is supposed to be small in the following sense: there exists a function $\varphi(\varepsilon)$ such that for any $p \geq 0$,

$$
\left\|P_{\varepsilon}(f)\right\|_{H_{\mathrm{per}}^{p}} \leq \varphi(\varepsilon)
$$

where $\varphi(\varepsilon)$ depends on $\|f\|_{H_{\mathrm{per}}^{p+k}}$ for some $k>0$, and goes to zero as $\varepsilon$ goes to zero.

Finally, in order to prove global existence with uniform regularity bounds, we shall require additional assumptions on the relation between the equilibrium distributions of the perturbed and unperturbed (periodized) Boltzmann equations, as well as the stability of the unperturbed equation (see the following statement).

Let us therefore write the unperturbed equation for reference:

$$
\left\{\begin{array}{l}
\frac{\partial f}{\partial t}=Q^{R}(f, f), \quad v \in \mathcal{D}_{L}, \quad t>0, \\
f(0, v)=f_{0}(v), \quad v \in \mathcal{D}_{L} .
\end{array}\right.
$$

Let us state the general stability theorem:

Theorem 3.1. Let us consider a perturbed Boltzmann equation (3.17) in the torus $\mathcal{D}_{L}$, where $Q^{R}$ is defined by (3.13) or (3.16), and for a sequence of smooth balanced perturbations $\left(P_{\varepsilon}=P_{\varepsilon}(f)\right)_{\varepsilon>0}$ which satisfy (3.18)-(3.20).

Assume that the constant functions are equilibria of the perturbed equation (3.17) (as for equation (3.21)) and that they are non-linearly locally stable in any $H_{\mathrm{per}}^{k}\left(\mathcal{D}_{L}\right)$ for equation (3.21).

We assume that $f_{0}$ is a non-negative function, non-zero everywhere, belonging to $H_{\mathrm{per}}^{k}\left(\mathcal{D}_{L}\right)$ with $k \in \mathbb{N}$ and $k>d / 2$. We consider a sequence of smooth balanced perturbations $f_{0, \varepsilon}$ of the initial datum for the perturbed problem (3.17) (not necessarily positive) such that

$$
\int_{\mathcal{D}_{L}} f_{0, \varepsilon}=\int_{\mathcal{D}_{L}} f_{0} \quad \text { and } \quad\left\|f_{0}-f_{0, \varepsilon}\right\|_{H_{\mathrm{per}}^{k}} \leq \psi(\varepsilon),
$$

where $\psi(\varepsilon)$ goes to zero when $\varepsilon$ goes to zero.

Then there exists $\varepsilon_{0}>0$ depending only on the collision kernel $B$, the truncation $R$, the constants in (3.19)-(3.20) for the perturbation, and the $L^{1}\left(\mathcal{D}_{L}\right)$ and $H_{\mathrm{per}}^{k}\left(\mathcal{D}_{L}\right)$ norms on $f_{0}$, such that for any $\varepsilon \in\left(0, \varepsilon_{0}\right)$,

(i) there exists a unique global smooth solution $f_{\varepsilon}$ to (3.17);

(ii) for any $p<k$, this solution belongs to $H_{\mathrm{per}}^{p}\left(\mathcal{D}_{L}\right)$ for all times with uniform bounds as time goes to infinity;

(iii) this solution remains "essentially non-negative" uniformly in time, in the sense that there is $\eta(\varepsilon)>0$ (with $\eta(\varepsilon) \rightarrow 0$ as $\varepsilon$ goes to 0 ) such that the non-positive part is $\eta(\varepsilon)$-small:

$$
\forall t \geq 0, \quad\left\|f_{\varepsilon}^{-}(t, \cdot)\right\|_{L^{\infty}} \leq \eta(\varepsilon),
$$

where $f_{\varepsilon}^{-}$denotes $\left|f_{\varepsilon}\right| \mathbf{1}_{\left\{f_{\varepsilon} \leq 0\right\}}$;

(iv) this solution $f_{\varepsilon}$ converges in $H_{\mathrm{per}}^{p}\left(\mathcal{D}_{L}\right)$ for any $p<k$, uniformly on any $[0, T], T>0$, to the solution $f$ of the unperturbed equation (3.21) when the parameter $\varepsilon$ goes to zero;

$(v)$ the solution $f_{\varepsilon}$ to (3.17) converges in $H_{\mathrm{per}}^{k}\left(\mathcal{D}_{L}\right)$ as time goes to infinity to the constant equilibrium distribution in the torus prescribed by its mass, and it is "asymptotically uniformly positive", that is, for $t$ larger than some fixed explicit time. 
We split the proof into two main steps: first in Section 4 we prove existence, uniqueness and smoothness of a solution on an arbitrary bounded time interval (as the size of perturbation goes to 0). The main difficulty is to prove that nonnegativity of the distribution function is recovered in a certain sense. Then, in Section 5 we study the asymptotic behavior and establish global stability in time. The main issue there is to prove regularizing properties of the gain operator $Q^{R,+}(f, f)$ and entropy production estimates on $Q^{R}$. Finally in Section 6 we apply the previous general results to spectral methods, and we prove their stability and convergence.

\section{Proof of Stability on an arbitrary bounded time interval}

In this section we first give some technical lemmas and next establish a result showing existence and uniqueness of a smooth solution on an arbitrary time interval to the perturbed equation (3.17), depending on an assumption of smallness on the size of the perturbation. Then, in Lemma 4.5 we prove the control of negative values of $f(t)$.

4.1. Preliminary results. We start this section by a classical result of $L^{p}$ estimates on the Boltzmann operator $Q^{R}(g, h)$ given by

$$
Q^{R}(g, h):=\int_{\mathcal{C}_{R}} \mathcal{B}(y, z)\left(g_{\star}^{\prime} h^{\prime}-g_{\star} h\right) d y d z .
$$

Lemma 4.1. Let the collision kernel $B$ satisfy the assumptions (2.2)-(2.4). Then, the periodized Boltzmann operator $Q^{R}$ (defined by (3.13) or (3.16) ) satisfies the following: for all $p \in[0, \infty]$ there exists a constant $C_{p}(R, B)>0$ such that

$$
\left\|Q^{R}(g, h)\right\|_{L^{p}},\left\|Q^{R}(h, g)\right\|_{L^{p}} \leq C_{p}(R, B)\|g\|_{L^{1}}\|h\|_{L^{p}} .
$$

Proof. The proof is exactly similar to the case of the usual Boltzmann collision operator for a collision kernel bounded with compact support; see for instance [20] for a recent proof.

Now, we prove smoothness of the solution to the perturbed problem (3.17) on a fixed time interval under the assumption of an a priori bound on the $L^{1}$ norm of the solution.

Lemma 4.2. Let us consider a collision kernel $B$ which satisfies the assumptions (2.2) -(2.4) and a sequence of smooth balanced perturbations $\left(P_{\varepsilon}=P_{\varepsilon}\left(f^{\varepsilon}\right)\right)_{\varepsilon>0}$ which satisfy (3.18)-(3.20), and let $T>0$ be the length of the time interval. Assume that $f_{0} \in H^{k}\left(\mathcal{D}_{L}\right)$ for $k \in \mathbb{N}$ and that $f(t)$ is a (not necessarily positive) solution to (3.17) with initial datum $f_{0}$, which satisfies the $L^{1}$-estimate

$$
\forall t \in[0, T], \quad\|f(t)\|_{L^{1}} \leq M .
$$

Then, there exists a constant $C_{k}(M)>0$, only depending on $M, R, T$ and $\left\|f_{0}\right\|_{H_{\mathrm{per}}^{k}}$, such that

$$
\forall t \in[0, T], \quad\|f(t)\|_{H_{\mathrm{per}}^{k}} \leq C_{k}(M) .
$$

Proof of Lemma 4.2. We proceed by induction on $k \geq 0$. For the first stage $k=0$, we apply Lemma 4.1 with $p=2$ and $g=h=f$ and we use assumption (3.19) on 
the perturbation

$$
\begin{aligned}
\frac{1}{2} \frac{d}{d t}\|f(t)\|_{L^{2}}^{2} & \leq\left\|Q^{R}(f, f)+P_{\varepsilon}(f)\right\|_{L^{2}}\|f(t)\|_{L^{2}} \\
& \leq\left(C_{2}(R, B)+C_{0}\right)\|f(t)\|_{L^{1}}\|f(t)\|_{L^{2}}^{2} .
\end{aligned}
$$

Hypothesis (4.2) provides a control on $\|f(t)\|_{L^{1}}$, and we can apply Gronwall's lemma to get the result (4.3) at stage $k=0$.

Let us now assume that (4.3) holds at stage $k \geq 0$, and let us prove that it also holds at stage $(k+1)$.

Let us first recall a formula on the derivatives of $Q^{R}$ : from the bilinearity of $Q^{R}$ and the translation invariance property of the periodized Boltzmann collision operator, one has

$$
\nabla_{v} Q^{R}(f, f)=Q^{R}\left(\nabla_{v} f, f\right)+Q^{R}\left(f, \nabla_{v} f\right)
$$

which yields a Leibniz formula at any order $s \in \mathbb{N}$ :

$$
\begin{aligned}
\left\|Q^{R}(f, f)\right\|_{H_{\mathrm{per}}^{s}}^{2} & =\sum_{|\nu| \leq s}\left\|\partial^{\nu} Q^{R}(f, f)\right\|_{L^{2}}^{2} \\
& \leq C \sum_{|\nu| \leq s} \sum_{|\mu| \leq|\nu|}\left(\begin{array}{c}
\nu \\
\mu
\end{array}\right)\left\|Q^{R}\left(\partial^{\mu} f, \partial^{\nu-\mu} f\right)\right\|_{L^{2}}^{2}
\end{aligned}
$$

Now, using (4.4) with $s=k+1$ we have

$$
\begin{aligned}
\left\|Q^{R}(f, f)\right\|_{H_{\mathrm{per}}^{k+1}}^{2} \leq C & \left\|Q^{R}(f, f)\right\|_{H_{\mathrm{per}}^{k}}^{2} \\
& +C \sum_{|\nu|=k+1} \sum_{|\mu| \leq k+1}\left(\begin{array}{c}
\nu \\
\mu
\end{array}\right)\left\|Q^{R}\left(\partial^{\mu} f, \partial^{\nu-\mu} f\right)\right\|_{L^{2}}^{2} .
\end{aligned}
$$

From Lemma 4.1 with $p=2$ and $g=\partial^{\mu} f, h=\partial^{\nu-\mu} f$, together with the hypothesis (4.3), we get

$$
\left\|Q^{R}(f, f)\right\|_{H_{\mathrm{per}}^{k}} \leq C(k, R)\|f\|_{H_{\mathrm{per}}^{k}}\|f\|_{H_{\mathrm{per}}^{k}} \leq C_{2}(k, R, B) C_{k}(M)^{2} .
$$

Then we split the last term of (4.5) in two parts for $\mu \neq 0$ and $\mu=0$. For $\mu \neq 0$, we again apply Lemma 4.1 with $p=2$ and $g=\partial^{\mu} f, h=\partial^{\nu-\mu} f$ and use the fact that both derivatives $|\mu|=\sum_{i=1}^{d}\left|\mu_{i}\right| \leq k$ and $|\nu-\mu| \leq k$ to obtain

$$
\sum_{|\nu|=k+1} \sum_{\substack{|\mu| \leq k+1 \\
\mu \neq 0}}\left(\begin{array}{c}
\nu \\
\mu
\end{array}\right)\left\|Q^{R}\left(\partial^{\mu} f, \partial^{\nu-\mu} f\right)\right\|_{L^{2}}^{2} \leq C_{3}(k, R, B) C_{k}(M)^{2} .
$$

Finally, for $\mu=0$ we apply Lemma 4.1 with $p=2$ and $g=f, h=\partial^{\nu} f$ to get

$$
\sum_{|\nu|=k+1}\left\|Q^{R}\left(f, \partial^{\nu} f\right)\right\|_{L^{2}}^{2} \leq C_{4}(k, R, B)\|f\|_{L^{1}}\|f\|_{H_{\mathrm{per}}^{k+1}} .
$$

Then, gathering inequalities from (4.6) to (4.8) and using the assumption (3.19) on the smooth balanced perturbation $P_{\varepsilon}$, we have

$$
\begin{aligned}
\frac{1}{2} \frac{d}{d t}\|f\|_{H_{\mathrm{per}}^{k+1}}^{2} & \leq\left\|Q^{R}(f, f)+P_{\varepsilon}(f)\right\|_{H_{\mathrm{per}}^{k+1}}\|f\|_{H_{\mathrm{per}}^{k+1}} \\
& \leq C\left(C_{2}+C_{3}\right) C_{k}(M)^{2}\|f\|_{H_{\mathrm{per}}^{k+1}}+C\left(C_{4}+C\right)\|f\|_{L^{1}}\|f\|_{H_{\mathrm{per}}^{k+1}}^{2} .
\end{aligned}
$$


Finally, using the control (4.2) on $\|f(t)\|_{L^{1}}$ we apply Gronwall's lemma to get (4.3) at stage $k+1$ : there exists a constant $C_{k+1}(M)$, only depending on $M, R, T$ and $\left\|f_{0}\right\|_{H_{\mathrm{per}}^{k+1}}^{k+1}$ such that

$$
\forall t \in[0, T], \quad\|f(t)\|_{H_{\mathrm{per}}^{k+1}} \leq C_{k+1}(M) .
$$

Then, we establish existence and uniqueness of a smooth solution for the perturbed problem (3.17) on a small time interval $[0, \bar{\tau}], \bar{\tau}>0$.

Proposition 4.3. Let us consider a collision kernel $B$ which satisfies the assumptions (2.2) -(2.4) and a sequence of smooth balanced perturbations $\left(P_{\varepsilon}=P_{\varepsilon}(f)\right)_{\varepsilon>0}$ which satisfy (3.18) -(3.20). We assume that $f_{0} \in H_{\mathrm{per}}^{k}\left(\mathcal{D}_{L}\right)$ for $k \in \mathbb{N}$ and set

$$
M=2\left\|f_{0}\right\|_{L^{1}} .
$$

Then there exists $\bar{\tau}=\bar{\tau}(M)>0$ such that for all $\varepsilon>0$ the perturbed Boltzmann equation (3.17) admits a unique solution (not necessarily positive) on the time interval $[0, \bar{\tau}]$, where the solution $f$ satisfies

$$
\forall t \in[0, \bar{\tau}], \quad\|f(t)\|_{L^{1}} \leq M .
$$

Moreover, there exists a constant $C_{k}(M)>0$, only depending on $M, R$, and $\left\|f_{0}\right\|_{H_{\mathrm{per}}^{k}}$, such that

$$
\forall t \in[0, \bar{\tau}], \quad\|f(t)\|_{H_{\mathrm{per}}^{k}} \leq C_{k}(M) .
$$

Proof of Proposition 4.3. First, we apply Lemma 4.1 with $p=1$ and $g=h=f$ to obtain

$$
\left\|Q^{R}(f, f)\right\|_{L^{1}} \leq C_{1}(R, B)\|f\|_{L^{1}}\|f\|_{L^{1}} .
$$

Moreover, using assumption (3.19) on the perturbation, there exists $C>0$ such that for all $\varepsilon>0$,

$$
\left\|P_{\varepsilon}(f)\right\|_{L^{1}} \leq C\|f\|_{L^{1}}^{2} .
$$

Therefore, we obtain a constant $C>0$, only depending on $R$ and the collision kernel $B$, such that

$$
\frac{d}{d t}\|f(t)\|_{L^{1}} \leq C\|f\|_{L^{1}}^{2}
$$

This implies that

$$
\|f(t)\|_{L^{1}} \leq \frac{\left\|f_{0}\right\|_{L^{1}}}{1-C\left\|f_{0}\right\|_{L^{1}} t} .
$$

Now, setting $M=2\left\|f_{0}\right\|_{L^{1}}$ and from the latter inequality, we show that there exists $\bar{\tau}<1 /\left(2 C\left\|f_{0}\right\|_{L^{1}}\right)$ such that

$$
\forall t \in[0, \bar{\tau}], \quad\|f(t)\|_{L^{1}} \leq M,
$$

which gives (4.10).

From the estimate in $L^{1}\left(\mathcal{D}_{L}\right)$ on the function $f(t)$ on the time interval $[0, \bar{\tau}]$, we prove existence and uniqueness of a solution by the Cauchy-Lipschitz theorem in $L^{1}\left(\mathcal{D}_{L}\right)$ (because of the truncation on $\mathcal{D}_{L}$ the collision kernel is a bounded bilinear function from $L^{1}\left(\mathcal{D}_{L}\right) \times L^{1}\left(\mathcal{D}_{L}\right)$ to $\left.L^{1}\left(\mathcal{D}_{L}\right)\right)$. Finally, from the bound (4.10) and the smoothness assumption $f_{0} \in H_{\mathrm{per}}^{k}\left(\mathcal{D}_{L}\right)$ on the initial datum, we are able to apply 
Lemma 4.1, which proves that there exists a constant $C_{k}(M)>0$, only depending on $M, R, T$ and $\left\|f_{0}\right\|_{H_{\mathrm{per}}^{k}}$, such that

$$
\|f(t)\|_{H_{\mathrm{per}}^{k}} \leq C_{k}(M)
$$

This concludes the proof.

By iterating Proposition 4.3 and Lemma 4.1 we observe that uniform control on the $L^{1}\left(\mathcal{D}_{L}\right)$ norm on an arbitrarily large time interval $[0, T]$, together with smoothness on the initial datum, will ensure existence and uniqueness of a smooth solution on this time interval $[0, T]$. Furthermore, we observe that the control on the $L^{1}\left(\mathcal{D}_{L}\right)$ norm is obvious for the classical Boltzmann equation, thanks to the positivity and mass preservations. Therefore, we shall now focus on the control of positivity of the solution, showing that the solution remains "almost positive" for an arbitrarily large time interval, hence allowing us to produce uniform control on the $L^{1}\left(\mathcal{D}_{L}\right)$ norm for an arbitrarily large time interval.

We first state a technical lemma which takes advantage of the mixing property of $Q^{R,+}$ in order to show spreading of the support of a characteristic function of a ball.

Lemma 4.4. Let us consider a collision kernel $B$ which satisfies the assumptions (2.2) -(2.4) and a truncated operator $Q^{R}$ defined by (3.13) or (3.16). Then for all $0<r<\sqrt{2} L$ we have

$$
Q^{R,+}\left(\mathbf{1}_{\mathcal{B}(v, r)}, \mathbf{1}_{\mathcal{B}(v, r)}\right) \geq C_{0} \mathbf{1}_{\mathcal{B}(v, \mu r)}
$$

for some explicit $\mu=\mu(R, L)>1$ and $C_{0}>0$.

Remark. Note that for $r \geq \sqrt{2} L$ one has $\mathbf{1}_{\mathcal{B}(v, r)}=1$ on the torus $[-L, L]^{d}$, and there is nothing to prove.

Proof of Lemma 4.4. The invariance by translation allows us to reduce the proof to the case $v=0$. The invariance by rotation implies that $I_{r}:=Q^{R,+}\left(\mathbf{1}_{\mathcal{B}(0, r)}, \mathbf{1}_{\mathcal{B}(0, r)}\right)$ is radially symmetric. More precisely, taking a $\mathcal{C}^{\infty}$ radially symmetric function $\phi$ such that $\phi>0$ on $\mathcal{B}=\mathcal{B}(0, r)$ and $\phi \leq \mathbf{1}_{\mathcal{B}}$ on $\mathbb{R}^{d}$, we have

- the function $v \longrightarrow Q^{R,+}(\phi, \phi)(v)$ is continuous,

- for all $v \in \mathbb{R}^{d}, I_{r}(v) \geq Q^{+}(\phi, \phi)(v)$,

- for all $v \in \mathcal{B}, Q^{R,+}(\phi, \phi)(v)>0$.

As a consequence, for any ball $\mathcal{B}^{\prime}=\mathcal{B}\left(0, r^{\prime}\right)$ strictly included in $\mathcal{B}$, there exists $\kappa_{r^{\prime}}>0$ such that $I_{r} \geq \kappa_{r^{\prime}} \mathbf{1}_{\mathcal{B}^{\prime}}$. $\mathcal{B}$.

In order to conclude, we just need to estimate the support of $I_{r}$ close to the ball

Let us fix $r^{\prime} \in(0, r)$ and choose $v^{\prime}, v_{\star}^{\prime} \in \mathcal{B}^{\prime}$ such that $\left|v^{\prime}\right|=\left|v_{\star}^{\prime}\right|=r^{\prime}$ and $\left|v^{\prime}-v_{\star}^{\prime}\right|=\min \left\{R ; \sqrt{2} r^{\prime}\right\}$. Then taking $\sigma$ in $\mathcal{B}^{\prime}$ such that $v, v_{\star}, v^{\prime}, v_{\star}^{\prime}$ is a square, we find $|v|=\sqrt{2} r^{\prime}$ if $\sqrt{2} r^{\prime} \leq R$ and

$$
|v|=\left[\left(r^{\prime}\right)^{2}-\frac{R^{2}}{4}\right]^{1 / 2}+\frac{R}{2}
$$

otherwise. We define

$\mu_{0}=\min _{R / \sqrt{2} \leq r^{\prime} \leq \sqrt{2} L}\left[1-\frac{R^{2}}{4\left(r^{\prime}\right)^{2}}\right]^{1 / 2}+\frac{R}{2 r^{\prime}}=\min _{R /(2 \sqrt{2} L) \leq y \leq 1 / \sqrt{2}}\left(\sqrt{1-y^{2}}+y\right) \in(1, \sqrt{2})$. 
The following concludes the proof: we deduce that for any $v$ such that $|v| \in$ $\left(r^{\prime}, \mu_{0} r^{\prime}\right)$ we have

$$
I_{r}(v) \geq Q^{+}(\phi, \phi)(v)>0
$$

since $\phi$ is strictly positive in the neighborhood of the $v^{\prime}, v_{\star}^{\prime}$ associated to $v$ constructed above. Hence we deduce by taking $r^{\prime}<r$ close to $r$ that for any $0<\mu<\mu_{0}$ we have

$$
I_{r} \geq C(\mu, r) \mathbf{1}_{\mathcal{B}(0, \mu r)}
$$

for some constant $C(\mu, r)>0$ depending continuously on $r$. We can choose $\mu=$ $\left(1+\mu_{0}\right) / 2$ for instance, and, for this choice of $\mu$, we take

$$
C_{0}=\min _{0 \leq r \leq \sqrt{2}} C(\mu, r)>0 .
$$

Finally, we establish the following positivity result on the solution to the perturbed problem (3.17):

Lemma 4.5. Let us consider a collision kernel $B$ which satisfies the assumptions (2.2) -(2.4), a truncated operator $Q^{R}$ defined by (3.13) or (3.16), and a sequence of smooth balanced perturbations $\left(P_{\varepsilon}=P_{\varepsilon}(f)\right)_{\varepsilon>0}$ which satisfy (3.18)-(3.20).

We assume that $f_{0}$ is a non-negative function such that $f_{0} \in H_{\mathrm{per}}^{k}\left(\mathcal{D}_{L}\right)$ with $k \in \mathbb{N}$ and $k>d / 2$. Moreover, we define $M=2\left\|f_{0}\right\|_{L^{1}}$ and $f_{0, \varepsilon}$ a smooth balanced perturbation of $f_{0}$, which is not necessarily positive and such that

$$
\int_{\mathcal{D}_{L}} f_{0, \varepsilon}=\int_{\mathcal{D}_{L}} f_{0} \quad \text { and } \quad\left\|f_{0}-f_{0, \varepsilon}\right\|_{H_{\mathrm{per}}^{k}} \leq \psi(\varepsilon),
$$

where $\psi(\varepsilon)$ goes to zero when $\varepsilon$ goes to zero. We also set $\bar{\tau}, C_{k}(M)>0$ as the constant defined in Lemma 4.1 such that from Proposition 4.3 we have

$$
\forall t \in[0, \bar{\tau}], \quad\|f(t)\|_{H_{\mathrm{per}}^{k}} \leq C_{k}(M) .
$$

Then there exists $\hat{\tau} \in(0, \bar{\tau})$ which only depends on $M, R$ and the collision kernel $B$, and there exists $\hat{\varepsilon}>0$ which only depends on $\hat{\tau}, C_{k}(M)$, such that for all $\varepsilon$ such that $0<\varepsilon<\hat{\varepsilon}$ and for any smooth solution $H_{\mathrm{per}}^{k}\left(\mathcal{D}_{L}\right)$ to the perturbed Boltzmann equation (3.17) with perturbed initial datum $f_{0, \varepsilon}$, we have

$$
\forall v \in \mathcal{D}_{L}, \quad f_{\varepsilon}(\hat{\tau}, v)>0 .
$$

Moreover there exists $\eta(\varepsilon)$, which goes to 0 as $\varepsilon$ goes to zero, such that the nonpositive part of $f$ satisfies

$$
\left\|f^{-}(t)\right\|_{L^{\infty}} \leq \eta(\varepsilon), \quad t \in[0, \hat{\tau}] .
$$

Proof of Lemma 4.5, Let $\bar{\tau}>0$ be the length of the time interval for which there exists a smooth solution to the perturbed Boltzmann equation (3.17) with perturbed initial datum $f_{0, \varepsilon}$ such that (in the following we omit the subscript $\varepsilon$ for the solution)

$$
\|f(t)\|_{H_{\mathrm{per}}^{k}} \leq C_{k}(M), \quad t \in[0, \bar{\tau}] .
$$

We split the proof into three steps: first, we give a classical estimate on the loss term $L^{R}(f)$, second we establish an estimate of $f^{-}$with respect to the amplitude of the perturbation $P_{\varepsilon}(f)$, and third we use the spreading properties of $Q^{R,+}(f, f)$ to prove that there exists $\hat{\tau} \in(0, \bar{\tau})$ such that

$$
f(\hat{\tau}, v)>0, \quad v \in \mathcal{D}_{L} .
$$


Step 1. Applying Proposition 4.3 for the control of $\|f(t)\|_{L^{1}}$ on the time interval $[0, \bar{\tau}]$, we get

$$
\left\|L^{R}(f)\right\|_{L^{\infty}} \leq C(R, B)\|f\|_{L^{1}} \leq C(R, B) M,
$$

which gives for all $t \in\left[0, \tau_{0}\right]$, with $\tau_{0}=\min \{\bar{\tau}, \ln 2 /(M C(R, B))\}$,

$$
2 \geq e^{M C(R, B) t} \geq e^{-\int_{0}^{t} L^{R}(f(s)) d s} \geq e^{-M C(R, B) t} \geq \frac{1}{2} .
$$

Step 2. Let us split $f$ as $f=f^{+}-f^{-}$, with $f^{ \pm}=\max \{0, \pm f\}$, and use the monotonicity of $Q^{R,+}$ for non-negative distribution functions. It follows that

$$
\begin{aligned}
Q^{R,+}(f, f) & =Q^{R,+}\left(f^{+}-f^{-}, f^{+}-f^{-}\right) \\
& \geq-\left[Q^{R,+}\left(f^{+}, f^{-}\right)+Q^{R,+}\left(f^{-}, f^{+}\right)\right] .
\end{aligned}
$$

On the time interval $\left[0, \tau_{0}\right]$, we apply Proposition 4.3 to estimate $\|f(t)\|_{L^{1}}$ and since $\left\|f^{+}(t)\right\|_{L^{1}} \leq\|f(t)\|_{L^{1}}$. We get from Lemma 4.1

$$
\left\|Q^{R,+}\left(f^{+}, f^{-}\right)\right\|_{L^{\infty}},\left\|Q^{R,+}\left(f^{-}, f^{+}\right)\right\|_{L^{\infty}} \leq C_{\infty}(R, B) M\left\|f^{-}(t)\right\|_{L^{\infty}},
$$

which yields using (4.15)

$$
Q^{R,+}(f, f) \geq-2 C_{\infty}(R, B) M\left\|f^{-}(t)\right\|_{L^{\infty}} .
$$

Thus from the Duhamel representation of the solution $f$, we have for $v \in \mathcal{D}_{L}$,

$$
\begin{aligned}
f(t, v)= & f_{0, \varepsilon}(v) e^{-\int_{0}^{t} L^{R}(f(s))(v) d s} \\
& +\int_{0}^{t}\left[Q^{R,+}(f(s), f(s))+P_{\varepsilon}(f(s))\right](v) e^{-\int_{s}^{t} L^{R}(f(u))(v) d u} d s \\
\geq & -2 \psi(\varepsilon)-\int_{0}^{t}\left[Q^{R,+}(f(s), f(s))+P_{\varepsilon}(f(s))\right](v) e^{-\int_{s}^{t} L^{R}(f(u))(v) d u} d s .
\end{aligned}
$$

Hence, we get from the lower estimate (4.17) of $Q^{R,+}(f, f)$ and the smallness assumption (3.20) of the perturbation $P_{\varepsilon}(f)$, for all $v \in \mathcal{D}_{L}$

$f^{-}(t, v)=\max \{0,-f(t, v)\} \leq 2 \psi(\varepsilon)+2 \int_{0}^{t}\left(2 C_{\infty}(R, B) M\left\|f^{-}(s)\right\|_{L^{\infty}}+\varphi(\varepsilon)\right) d s$.

Finally, we take the supremum in $v \in \mathcal{D}_{L}$ and apply Gronwall's lemma to get for any $0 \leq \tau \leq \tau_{0}$,

$$
\left\|f^{-}(t)\right\|_{L^{\infty}} \leq\left(2 \psi(\varepsilon)+2 \tau_{0} \varphi(\varepsilon)\right) e^{4 C_{\infty}(R, B) M t}:=\eta(\varepsilon), \quad t \in\left[0, \tau_{0}\right],
$$

which proves (4.12) on the time interval $\left[0, \tau_{0}\right]$.

Step 3. Let us prove that there exists $\hat{\tau} \in\left(0, \tau_{0}\right)$ such that

$$
f(\hat{\tau}, v)>0, \quad v \in \mathcal{D}_{L} .
$$

We start again with the Duhamel representation of the solution

$$
\begin{aligned}
f(t, v)= & f_{0, \varepsilon}(v) e^{-\int_{0}^{t} L^{R}(f(s))(v) d s} \\
& +\int_{0}^{t}\left[Q^{R,+}(f(s), f(s))+P_{\varepsilon}(f(s))\right](v) e^{-\int_{s}^{t} L^{R}(f(u))(v) d u} d s,
\end{aligned}
$$

but we now take into account the fact that the first term is essentially positive and we use the spreading property of the operator $Q^{R,+}$ (Lemma 4.4). 
On the one hand since the initial datum is smooth enough $(k>d / 2$ is large enough such that $f_{0, \varepsilon}$ is Hölder), there exists an explicit $\delta>0$ depending on $C_{k}(M)$ such that for $\varepsilon$ small enough, there exists $v_{0} \in \mathcal{D}_{L}$ such that

$$
f_{0, \varepsilon}(v) \geq \frac{\eta}{2} \mathbf{1}_{\mathcal{B}\left(v_{0}, \delta\right)}(v)-\psi(\varepsilon), \quad \text { with } \eta=\frac{\left\|f_{0}\right\|_{L^{1}}}{(2 L)^{d}} .
$$

On the other hand, using the lower bound on the gain operator (4.17) and the estimate (4.12) of $f^{-}(t)$. It gives

$$
Q^{R,+}(f, f) \geq-2 C_{\infty}(R, B) M \eta(\varepsilon) .
$$

Finally, using (4.14) and the smallness assumption on $P_{\varepsilon}(f)$, it first yields for any $0<\tau<\tau_{0}$,

$$
f(t, v) \geq A_{0} \mathbf{1}_{\mathcal{B}\left(v_{0}, \delta\right)}(v)-\varphi_{1}(\varepsilon)
$$

with

$$
A_{0}:=\frac{\eta}{4}, \quad \varphi_{0}(\varepsilon):=2 \tau_{0}\left(2 C_{\infty}(R, B) M \eta(\varepsilon)+\varphi(\varepsilon)\right)+\psi(\varepsilon) .
$$

Now we choose $\varepsilon_{1}>0$ small enough such that

$$
0<\frac{A_{0}}{2} \leq A_{0}-\varphi_{0}\left(\varepsilon_{1}\right) \quad \Longleftrightarrow \quad \varphi_{0}\left(\varepsilon_{1}\right) \leq \frac{A_{0}}{2} .
$$

Thus, we get on any time interval $[0, \tau] \subset\left[0, \tau_{0}\right]$

$$
f^{+}(t, v) \geq \frac{A_{0}}{2} \mathbf{1}_{\mathcal{B}\left(v_{0}, \delta\right)}(v) .
$$

Hence, using the spreading properties of the operator $Q^{R,+}(f, f)$ of Lemma 4.4 and the monotonicity of $Q^{R,+}$ for non-negative distribution functions, it follows that

$$
\begin{aligned}
Q^{R,+}\left(f^{+}, f^{+}\right) & \geq \frac{A_{0}^{2}}{4} Q^{R,+}\left(\mathbf{1}_{\mathcal{B}\left(v_{0}, \delta\right)}, \mathbf{1}_{\mathcal{B}\left(v_{0}, \delta\right)}\right) \\
& \geq \frac{A_{0}^{2}}{4} C_{0} \mathbf{1}_{\mathcal{B}\left(v_{0}, \mu \delta\right)} .
\end{aligned}
$$

Next, we again use the uniform bounds previously established in (4.16) on $Q^{R,+}\left(f^{+}, f^{-}\right)$and $Q^{R,+}\left(f^{-}, f^{+}\right)$:

$$
\begin{aligned}
Q^{R,+}(f, f) & \geq Q^{R,+}\left(f^{+}, f^{+}\right)-Q^{R,+}\left(f^{-}, f^{+}\right)-Q^{R,+}\left(f^{+}, f^{-}\right) \\
& \geq \frac{A_{0}^{2}}{4} C_{0} \mathbf{1}_{\mathcal{B}\left(v_{0}, \mu \delta\right)}-2 C_{\infty}(R, B) M\left(\eta(\varepsilon)+\varphi_{0}(\varepsilon)\right) .
\end{aligned}
$$

Finally, from the smallness assumption (3.20) of the perturbation $P_{\varepsilon}(f)$, it yields for $t \in[\tau / 2, \tau]$ and with (4.14),

$$
\begin{aligned}
f(t, v) & \geq-\psi(\varepsilon)+\int_{0}^{t}\left[Q^{R,+}(f(s), f(s))+P_{\varepsilon}(f(s))\right](v) e^{-\int_{s}^{t} L^{R}(f(u))(v) d u} d s \\
& \geq \frac{\tau}{2} \frac{A_{0}^{2}}{4} C_{0} \mathbf{1}_{\mathcal{B}\left(v_{0}, \mu \delta\right)}-2 \tau\left[2 C_{\infty}(R, B) M\left(\eta(\varepsilon)+\varphi_{0}(\varepsilon)\right)+\varphi(\varepsilon)\right]-\psi(\varepsilon) \\
& =A_{1} \mathbf{1}_{\mathcal{B}\left(v_{0}, \mu \delta\right)}-\varphi_{1}(\varepsilon)
\end{aligned}
$$

with

$$
A_{1}=\frac{\tau}{8} A_{0}^{2} C_{0}, \quad \varphi_{1}(\varepsilon):=2 \tau_{0}\left[2 C_{\infty}(R, B) M\left(\eta(\varepsilon)+\varphi_{0}(\varepsilon)\right)+\varphi(\varepsilon)\right]-\psi(\varepsilon) .
$$


Now, we proceed by induction: assume that there exists $\left(A_{j}, \varepsilon_{j}, \varphi_{j}\right)$ such that on the time interval $\left[\tau-\tau / 2^{j}, \tau\right] \subset\left[0, \tau_{0}\right]$ and for $\varepsilon \in\left(0, \varepsilon_{j}\right)$, we have

$$
f(t, v) \geq A_{j} \mathbf{1}_{\mathcal{B}\left(v_{0}, \mu^{j} \delta\right)}(v)-\varphi_{j}(\varepsilon),
$$

where

and $\varphi_{j}(\varepsilon) \rightarrow 0$ as $\varepsilon \rightarrow 0$.

$$
A_{j}:=\left(\frac{\tau}{8}\right)^{2^{j}-1} A_{0}^{2^{j}} C_{0}^{2^{j-1}-1}
$$

Using the same method as before, we first set $\varepsilon_{j+1}$ such that

$$
f^{+}(t, v) \geq \frac{A_{j}}{2} \mathbf{1}_{\mathcal{B}\left(v_{0}, \mu^{j} \delta\right)}
$$

and prove that for $t \in\left[\tau-\frac{\tau}{2^{j+1}}, \tau\right]$

$$
Q^{R,+}(f, f) \geq\left(\frac{A_{j}}{2}\right)^{2} C_{0} \mathbf{1}_{\mathcal{B}\left(v_{0}, \mu^{j+1} \delta\right)}-2 C_{\infty}(R, B) M\left(\eta(\varepsilon)+\varphi_{j}(\varepsilon)\right) .
$$

Then, from the Duhamel formula and the smallness assumption of $P_{\varepsilon}(f)$, we finally get the following lower bound:

$$
f(t, v) \geq A_{j+1} \mathbf{1}_{\mathcal{B}\left(v_{0}, \mu^{j+1} \delta\right)}-\varphi_{j+1}(\varepsilon),
$$

with

$A_{j+1}=\frac{\tau}{8} A_{j}^{2} C_{0}, \quad \varphi_{j+1}(\varepsilon):=2 \tau_{0}\left[2 C_{\infty}(R, B) M\left(\eta(\varepsilon)+\varphi_{j}(\varepsilon)\right)+\varphi(\varepsilon)\right]-\psi(\varepsilon)$.

Since $\mu>1$, the ball $\mathcal{B}\left(v_{0}, \mu^{j} \delta\right)$ eventually recovers the periodic box $[-L, L]^{d}$ i.e., for some $J$ large enough: $[-L, L]^{d} \subset \mathcal{B}\left(v_{0}, \mu^{J} \delta\right)$, and for all $t \in\left[\tau-\frac{\tau}{2^{J}}, \tau\right]$, by applying the $J$ 's times the previous induction we get for $\varepsilon \in\left(0, \varepsilon_{J}\right)$

$$
f(t, v) \geq A_{J} \mathbf{1}_{\mathcal{B}\left(v_{0}, \mu^{J} \delta\right)}(v)-\varphi_{J}(\varepsilon) .
$$

Finally, up to reducing $\varepsilon$ further, we have proved that there exists $(\hat{\tau}, \hat{\varepsilon})$ which only depends on the collision kernel $B$, the initial datum $f_{0}, L$ and the perturbation function $\varphi=\varphi(\varepsilon)$ such that for all $0<\varepsilon<\hat{\varepsilon}$,

$$
\forall v \in \mathcal{D}_{L}, \quad f(\hat{\tau}, v)>0 .
$$

\subsection{Existence and regularity on a bounded time interval.}

Proposition 4.6. Let us consider a fixed time $T>0$, a collision kernel $B$ which satisfies the assumptions (2.2) -(2.4), a truncation $Q^{R}$ defined by (3.13) or (3.16), and a sequence of smooth balanced perturbations $\left(P_{\varepsilon}=P_{\varepsilon}(f)\right)_{\varepsilon>0}$ which satisfy (3.18) - (3.20) .

We assume that $f_{0}$ is a non-negative function, not zero everywhere, and such that $f_{0} \in H_{\mathrm{per}}^{k}\left(\mathcal{D}_{L}\right)$ with $k \in \mathbb{N}$ and $k>d / 2$. We define $M=2\left\|f_{0}\right\|_{L^{1}}$ and $\left(f_{0, \varepsilon}\right)_{\varepsilon>0}$ a sequence of smooth perturbations of $f_{0}$ (which is not necessarily positive) such that

$$
\int_{\mathcal{D}_{L}} f_{0, \varepsilon}=\int_{\mathcal{D}_{L}} f_{0} \quad \text { and } \quad\left\|f_{0}-f_{0, \varepsilon}\right\|_{H_{\mathrm{per}}^{k}} \leq \psi(\varepsilon),
$$

where $\psi(\varepsilon)$ goes to zero when $\varepsilon$ goes to zero.

Then there exists $\hat{\varepsilon}>0$, which only depends on the $H_{\mathrm{per}}^{k}\left(\mathcal{D}_{L}\right)$ and $L^{1}\left(\mathcal{D}_{L}\right)$ norms of $f_{0}$, such that for all $\varepsilon \in(0, \hat{\varepsilon})$,

(i) there is a unique smooth solution $f_{\varepsilon}=f_{\varepsilon}(t, \cdot)$ on $[0, T]$ to the perturbed equation (3.17) with initial datum $f_{0, \varepsilon}$;

(ii) this belongs to $H_{\mathrm{per}}^{k}\left(\mathcal{D}_{L}\right)$ (with bound growing at most exponentially); 
(iii) there is some explicit $\eta(\varepsilon)>0$ (with $\eta(\varepsilon) \rightarrow 0$ as $\varepsilon$ goes to 0 ) such that the non-positive part is $\eta(\varepsilon)$-small:

$$
\forall t \in[0, T], \quad\left\|f_{\varepsilon}^{-}(t, \cdot)\right\|_{L^{\infty}} \leq \eta(\varepsilon),
$$

where $f_{\varepsilon}^{-}$denotes $\max \left\{0,-f_{\varepsilon}\right\}$;

(iv) this solution satisfies for any $p<k$

$$
\forall t \in[0, T], \quad\left\|f(t, \cdot)-f_{\varepsilon}(t, \cdot)\right\|_{H_{\mathrm{per}}^{p}} \leq \bar{\varphi}(\varepsilon),
$$

where $f(t, \cdot)$ is the solution of unperturbed periodized Boltzmann equation (3.21) and $\bar{\varphi}(\varepsilon)$ is another explicit function which goes to zero as $\varepsilon$ goes to zero. Hence up to reducing $\varepsilon$, the perturbed solution remains close to the unperturbed solution on the finite time interval on which we have constructed.

Proof. We set

$$
M_{k}(T):=\left\|f_{0}\right\|_{H_{\mathrm{per}}^{k}} e^{C M T} .
$$

First, applying Proposition 4.3 we have proven that there exists a small $\bar{\tau}>0$ such that the perturbed Boltzmann equation (3.17) admits a unique smooth solution on the time interval $[0, \bar{\tau}]$ with

$$
\left\|f_{\varepsilon}(t)\right\|_{L^{1}} \leq M
$$

and

$$
\left\|f_{\varepsilon}(t)\right\|_{H_{\mathrm{per}}^{k}} \leq M_{k}(\bar{\tau}) \leq M_{k}(T) .
$$

Moreover, from Lemma 4.5 there exist $\hat{\tau} \leq \bar{\tau}$ and $\hat{\varepsilon}>0$, only depending on $M$, $M_{k}(\bar{\tau})<M_{k}(T), R$ and the collision kernel $B$, such that for all $0<\varepsilon<\hat{\varepsilon}$,

$$
\begin{gathered}
\forall v \in \mathcal{D}_{L}, \quad f_{\varepsilon}(\hat{\tau}, v)>0, \\
\left\|f^{-}(t)\right\|_{L^{\infty}} \leq \eta(\varepsilon), \quad t \in[0, \hat{\tau}],
\end{gathered}
$$

and

$$
\forall t \in[0, \hat{\tau}], \quad\|f(t)\|_{H_{\mathrm{per}}^{k}} \leq\left\|f_{0}\right\|_{H_{\mathrm{per}}^{k}} e^{C M 2 \hat{\tau}} .
$$

Then, from the preservation of mass under the action of $Q^{R}$ and $P_{\varepsilon}$,

$$
\int_{\mathcal{D}_{L}} Q^{R}\left(f_{\varepsilon}, f_{\varepsilon}\right)(v) d v=\int_{\mathcal{D}_{L}} P_{\varepsilon}\left(f_{\varepsilon}\right)(v) d v=0
$$

we have that

$$
\int_{\mathcal{D}_{L}} f_{\varepsilon}(\hat{\tau}, v) d v=\int_{\mathcal{D}_{L}} f_{0}(v) d v
$$

Since $f_{0}$ is a non-negative function, it gives that at time $\hat{\tau}$

$$
\left\|f_{\varepsilon}(\hat{\tau})\right\|_{L^{1}}=\left\|f_{0}\right\|_{L^{1}}
$$

and on the time interval $t \in[0, \hat{\tau}]$ we have

$$
\left\|f_{\varepsilon}(t)\right\|_{L^{1}} \leq M \quad \text { and }\left\|f_{\varepsilon}(t)\right\|_{H_{\mathrm{per}}^{k}} \leq M_{k}(T) .
$$

Therefore, we consider the perturbed Boltzmann equation (3.17) starting from $f_{\varepsilon}(\hat{\tau})$ as initial data. On the time interval $[\hat{\tau}, 2 \hat{\tau}]$ we apply Proposition 4.3 and get that

$$
\left\|f_{\varepsilon}(t)\right\|_{L^{1}} \leq 2\left\|f_{\varepsilon}(\hat{\tau})\right\|_{L^{1}}=2\left\|f_{0}\right\|_{L^{1}}=M, \quad \forall t \in[\hat{\tau}, 2 \hat{\tau}]
$$


and

$$
\begin{aligned}
\left\|f_{\varepsilon}(t)\right\|_{H_{\mathrm{per}}^{k}} & \leq\left\|f_{\varepsilon}(\hat{\tau})\right\|_{H_{\mathrm{per}}^{k}} e^{C M(2 \hat{\tau}-\hat{\tau})} \\
& \leq\left\|f_{0}\right\|_{H_{\mathrm{per}}^{k}} e^{C M 2 \hat{\tau}} \\
& \leq\left\|f_{0}\right\|_{H_{\mathrm{per}}^{k}} e^{C M T}=M_{k}(T), \quad \forall t \in[\hat{\tau}, 2 \hat{\tau}] .
\end{aligned}
$$

Moreover, since $\hat{\tau}$ only depends on $M, M_{k}(T), B$ and $R$, we can again apply Lemma 4.5 on the time interval $[\hat{\tau}, 2 \hat{\tau}]$, which yields that

$$
f_{\varepsilon}(2 \hat{\tau})>0 \text {. }
$$

We finally proceed by induction to prove existence and uniqueness of a smooth solution $f_{\varepsilon}$ of the perturbed Boltzmann equation (3.17) on the time interval $[0, T]$, which proves assertions $(i),(i i)$ and $(i i i)$.

To prove $(i v)$, we compute the difference between the solution $f(t)$ to the unperturbed problem (3.21) and the solution $f_{\varepsilon}(t)$ to (3.17):

$$
\frac{\partial\left(f-f_{\varepsilon}\right)}{\partial t}=\frac{1}{2}\left(Q^{R}\left(f-f_{\varepsilon}, f+f_{\varepsilon}\right)+Q^{R}\left(f+f_{\varepsilon}, f-f_{\varepsilon}\right)\right)+P_{\varepsilon}\left(f_{\varepsilon}\right) .
$$

Then, using the smoothness of $f$ and $f_{\varepsilon}$, we have from Lemma 4.1 for any $p<k$ that

$\left\|Q^{R}\left(f-f_{\varepsilon}, f+f_{\varepsilon}\right)\right\|_{H_{\mathrm{per}}^{p}},\left\|Q^{R}\left(f+f_{\varepsilon}, f-f_{\varepsilon}\right)\right\|_{H_{\mathrm{per}}^{p}} \leq C_{p}(M)\left\|f+f_{\varepsilon}\right\|_{H_{\mathrm{per}}^{p}}\left\|f-f_{\varepsilon}\right\|_{H_{\mathrm{per}}^{p}}$, and since the perturbation is small (assumption (3.20)),

$$
\left\|P_{\varepsilon}(f)\right\|_{H_{\mathrm{per}}^{p}} \leq \varphi(\varepsilon)
$$

it yields that for all $t \in[0, T]$

$$
\left\|f(t)-f_{\varepsilon}(t)\right\|_{H_{\mathrm{per}}^{p}} \leq \bar{\varphi}(\varepsilon)
$$

for some function $\bar{\varphi}(\varepsilon)$ going to zero as $\varepsilon$ goes to zero.

\section{Asymptotic Behavior and gLobal in time Stability}

In this section we shall study the asymptotic behavior of the (unperturbed) periodized Boltzmann equation (3.21) based on a regularity study (in the spirit of [20]) and the entropy - entropy production theory (mainly relying on the method developed in [34]). Finally, on the basis of these results we shall prove a global in times stability result for the perturbed equation (3.17).

5.1. Regularity study of the periodized Boltzmann equation. Let us prove the following result

Proposition 5.1. Let us consider $0 \leq f_{0} \in L^{1}\left(\mathcal{D}_{L}\right)$ such that $f_{0} \in H_{\mathrm{per}}^{k}\left(\mathcal{D}_{L}\right)$ for some $k \geq 0$. Then there is a constant $C>0$ depending on the $L^{1}$ and $H_{\mathrm{per}}^{k}\left(\mathcal{D}_{L}\right)$ norms of $f_{0}$ such that the unique global non-negative solution $(f(t))_{t \geq 0}$ to the periodized equation (3.21) satisfies

$$
\forall t \geq 0, \quad\|f(t)\|_{H_{\mathrm{per}}^{k}} \leq C .
$$

We proceed as in 20]. In particular, we shall extend Lions' regularity result on $Q^{+}$to the truncated case $Q^{R,+}[16,17$. Hence we shall first prove the regularity property on the gain operator when the collision kernel is smooth and compactly supported, avoiding cancellations at zero relative velocities. Then, we shall include the non-smooth part of the kernel using the loss operator. 
We shall split the collision kernel into a smooth and a non-smooth part. As a convention, we shall use subscripts "s" for smooth and "ns" for the non-smooth parts. In terms of the classical truncation (3.13) we set

$$
\left\{\begin{array}{l}
\mathcal{B}_{s}^{R}(|g|, \cos \theta)=\mathcal{B}(|g|, \cos \theta) \chi_{\eta}^{R}(|g|) \zeta_{\eta}(|g|) \Theta_{\eta}(\cos \theta) \\
\mathcal{B}_{\mathrm{ns}}^{R}(|g|, \cos \theta)=\mathcal{B}^{R}(|g|, \cos \theta)-\mathcal{B}_{s}^{R}(|g|, \cos \theta)
\end{array}\right.
$$

where $\chi_{\eta}^{R}(g)$ is the mollified $\mathscr{C}^{\infty}$ version of $\mathbf{1}_{|g| \leq R}, \zeta_{\eta}(g)$ is the mollified $\mathscr{C}^{\infty}$ version of $\mathbf{1}_{|g| \geq \eta}$, and $\Theta_{\eta}$ is a $\mathscr{C}^{\infty}$ function on $[-1,1]$ which is 1 on $-1+2 \eta \leq u \leq 1-2 \eta$, and 0 in $[-1,-1+\eta)$ and $(1-\eta, 1]$ (the parameter $\eta$ is the mollification parameter).

In terms of the "fast" truncation (3.16) we set

$$
\left\{\begin{array}{l}
\mathcal{B}_{s}^{R}(|y|,|z|)=\mathcal{B}(|y|,|z|) \chi_{\eta}^{R}(|z|) \chi_{\eta}^{R}(|y|) \zeta_{\eta}(|z+y|) \Theta_{\eta}\left(\frac{|y|}{\sqrt{|y|^{2}+|z|^{2}}}\right) \\
\mathcal{B}_{\mathrm{ns}}^{R}(|y|,|z|)=\mathcal{B}^{R}(|y|,|z|)-\mathcal{B}_{s}^{R}(|y|,|z|),
\end{array}\right.
$$

with the same notation.

We deduce the following decomposition of the collision operator:

$$
Q^{R,+}=Q_{\mathrm{s}}^{R,+}+Q_{\mathrm{ns}}^{R,+},
$$

where for instance, with the variables from the "fast" truncation,

$$
Q_{\mathrm{s}}^{R,+}(f, f)=\int_{y \in \mathbb{R}^{d}} \int_{z \in \mathbb{R}^{d}} \mathcal{B}_{\mathrm{s}}^{R}(|y|,|z|) \delta(y \cdot z) f(v+z) f(v+y) d y d z .
$$

Under the assumption that both $\Phi$ and $b$ defined in (2.2) are smooth, the regularized truncature introduced above ensures that there exist two functions $\Phi_{\eta}^{R}$ and $b_{\eta}^{R}$ such that

$$
\left\{\begin{array}{l}
B_{\eta}^{R}(|z|, \cos \theta)=\Phi_{\eta}^{R}(|z|) b_{\eta}^{R}(\cos \theta) \\
\Phi_{\eta}^{R} \in \mathscr{C}_{0}^{\infty}\left(\mathbb{R}^{d} \backslash\{0\}, \mathbb{R}\right), \quad b_{\eta}^{R} \in \mathscr{C}_{0}^{\infty}([-1,1], \mathbb{R})
\end{array}\right.
$$

In the following lemma we shall prove the regularity property of $Q_{\mathrm{s}}^{R,+}$.

Lemma 5.2. Let $B_{\eta}^{R}\left(\left|v-v_{\star}\right|, \cos \theta\right)$ satisfy the assumption (2.2) $-(2.4)$ and (5.2). Then, for all $r \in \mathbb{R}^{+}$

$$
\left\|Q_{\mathrm{s}}^{R,+}(f, f)\right\|_{H_{\mathrm{per}}^{r+\frac{d-1}{2}}} \leq C_{\mathrm{reg}}\left(r, \mathcal{B}_{\mathrm{s}}^{R}\right)\|f\|_{L^{1}}\|f\|_{H_{\mathrm{per}}^{r}}
$$

where the constant $C_{\mathrm{reg}}\left(r, \mathcal{B}_{\mathrm{s}}^{R}\right)$ depends only on $r$ and on the collision kernel.

Proof. We closely follow the proof given by Lions [16, 17], and simplified and then reformulated in [35, 20]. Again the preservation of the translation invariance by the truncation is fundamental. Starting from the collision operator in the form (5.1) and performing a change of variable we get for $v \in \mathcal{D}_{L}$

$Q_{\mathrm{s}}^{R,+}(f, f)=\int_{\mathbb{R}^{d} \times \mathbb{R}^{d}} \tilde{\mathcal{B}}_{\mathrm{s}}^{R}\left(\left|v_{\star}^{\prime}-v^{\prime}\right|,\left|v^{\prime}-v\right|\right) \delta\left(\left(v_{\star}^{\prime}-v\right) \cdot\left(v^{\prime}-v\right)\right) f\left(v_{\star}^{\prime}\right) f\left(v^{\prime}\right) d v_{\star}^{\prime} d v^{\prime}$, where $\tilde{\mathcal{B}}_{\mathrm{s}}^{R}$ only depends on $\mathcal{B}_{\mathrm{s}}^{R}$. Then we set 36

$$
T g(y)=\int_{y+y^{\perp}} \tilde{\mathcal{B}}_{\mathrm{s}}^{R}(|z|,|y|) g(z) d z, \quad \tau_{z} g(\cdot)=g(\cdot-z),
$$

where

$$
y^{\perp}=\left\{z \in \mathbb{R}^{d}, \quad z^{t} \cdot y=0\right\},
$$


and we easily get for $v \in \mathcal{D}_{L}$

$$
Q_{\mathrm{s}}^{R,+}(f, f)=\int_{\mathbb{R}^{d}} f\left(v^{\prime}\right)\left(\tau_{v^{\prime}} \circ T \circ \tau_{-v^{\prime}}\right) f(v) d v^{\prime} .
$$

Now, we want to estimate Sobolev norms of $Q_{\mathrm{s}}^{R,+}$ as a function defined in the torus $\mathcal{D}_{L}$. Applying the Fubini theorem with the discrete and Lebesgue measures and the Cauchy-Schwarz inequality, it leads us to estimate the Sobolev norms of the Radon transform $T$ on the torus $\mathcal{D}_{L}$ :

$$
\left\|Q_{\mathrm{s}}^{R,+}(f, f)\right\|_{H_{\mathrm{per}}^{r+\frac{d-1}{2}}}^{2} \leq\|f\|_{L^{1}} \int_{\mathbb{R}^{d}}\left|f\left(v^{\prime}\right)\right|\left\|\tau_{v^{\prime}} \circ T \circ \tau_{-v^{\prime}} f\right\|_{H_{\mathrm{per}}^{r+\frac{d-1}{2}}}^{2} d v^{\prime} .
$$

On the one hand, since the kernel $\tilde{\mathcal{B}}_{\mathrm{s}}^{R}$ is compactly supported in $y$ and $z$, the operator $T$ maps periodic functions $g$ to a compactly supported function $T g$ with $\operatorname{supp}(T g) \subset \mathcal{B}_{R} \subset \mathcal{D}_{L}$. Then we can consider $T g$ as a function in the whole space $\mathbb{R}^{d}$.

On the other hand, using the regularized truncations $\chi_{\eta}^{R}$ and $\Theta_{\eta}$ and the smoothness of $\Phi$ and $b$ in (2.2), it yields that $B_{\eta}^{R}=\Phi_{\eta}^{R}\left(\left|v-v_{\star}\right|\right) b_{\eta}^{R}(\cos \theta)$ with

$$
\Phi_{\eta}^{R} \in \mathscr{C}_{o}^{\infty}(\mathbb{R}, \mathbb{R}), \quad b_{\eta}^{R} \in \mathscr{C}_{o}^{\infty}([-1,1], \mathbb{R}) .
$$

Then we can directly apply the result in [20, where the authors proved the following regularity estimates on the Radon transform $T$ for smooth kernels:

$$
\|T g\|_{H^{r+\frac{d-1}{2}}} \leq C_{\mathrm{reg}}\left(r, \mathcal{B}_{\mathrm{s}}^{R}\right)\|g\|_{H^{r}}
$$

for a function $g$ defined in $\mathbb{R}^{d}$. However, in the proof of the latter inequality, we can replace $g$ by the smooth and compactly supported function $g \chi_{\eta}^{R}$ for which $\operatorname{supp}\left(\chi_{\eta}^{R} g\right) \subset \mathcal{B}_{R} \subset \mathcal{D}_{L}$. Thus, for all $g$ defined in the torus $\mathcal{D}_{L}$, we get

$$
\|T g\|_{H_{\mathrm{per}}^{r+\frac{d-1}{2}}}=\|T g\|_{H^{r+\frac{d-1}{2}}} \leq C_{\mathrm{reg}}\left(r, \mathcal{B}_{\mathrm{s}}^{R}\right)\|g\|_{H_{\mathrm{per}}^{r}} .
$$

Finally gathering (5.3) and (5.4), we obtain the result

$$
\left\|Q_{\mathrm{s}}^{R,+}(f, f)\right\|_{H_{\mathrm{per}}^{r+\frac{d-1}{2}}} \leq C_{\mathrm{reg}}\left(r, \mathcal{B}_{\mathrm{s}}^{R}\right)\|f\|_{L^{1}}\|f\|_{H_{\mathrm{per}}^{r}} .
$$

Corollary 5.3. Let $B_{\eta}^{R}\left(\left|v-v_{\star}\right|, \cos \theta\right)$ satisfy the assumptions (2.2)-(2.4) and (5.2)). Then, for all $p \in(1, \infty)$

$$
\left\|Q_{\mathrm{s}}^{R,+}(f, f)\right\|_{L^{q}} \leq C_{\mathrm{reg}}\left(r, \mathcal{B}_{\mathrm{s}}^{R}\right)\|f\|_{L^{1}}\|f\|_{L^{p}}
$$

with

$$
q= \begin{cases}\frac{p}{2-\frac{1}{d}+p\left(\frac{1}{d}-1\right)} & \text { if } p \in(1,2], \\ p d & \text { if } p \in[2, \infty) .\end{cases}
$$

Proof. It is a direct consequence of Sobolev embedding and interpolation between $L^{p}$ spaces.

Now we extend the regularity of $Q^{R,+}$ to general non-smooth kernels.

Lemma 5.4. Let $B$ be a collision kernel satisfying (2.2)-(2.4). Then, for all $p>1$, there exist constants $C, \kappa$ and $q<p$ ( $q$ only depending on $p$ and $d$ ), such that for 
all $\delta>0$ and for all measurable functions $f$

$$
\left\|Q^{R,+}(f, f)\right\|_{L^{p}} \leq C \delta^{-\kappa}\|f\|_{L^{1}}\|f\|_{L^{q}}+\delta\|f\|_{L^{1}}\|f\|_{L^{p}}
$$

Proof. We use a decomposition approach and split the operator $Q^{R,+}$ as the sum of a smooth part and a non-smooth part:

$$
Q^{R,+}=Q_{\mathrm{s}}^{R,+}+Q_{\mathrm{ns}}^{R,+}
$$

where $Q_{\mathrm{s}}^{R,+}$ is given by (5.1). Then, applying Corollary 5.3 we have for all $p \in$ $(1, \infty)$ that there exist $q<p$, namely (the role of $q$ and $p$ are exchanged here with respect to Corollary 5.3 ,

$$
q= \begin{cases}\frac{(2 d-1) p}{d+(d-1) p} & \text { if } p \in(1,2 d] \\ \frac{p}{d} & \text { if } p \in[2 d, \infty)\end{cases}
$$

and $C_{\text {reg }}\left(\eta, \mathcal{B}_{\mathrm{s}}^{R}\right)$, depending on the the regularization parameter $\eta$ and blowing-up polynomially when $\eta \rightarrow 0$ such that

$$
\left\|Q_{\mathrm{s}}^{R,+}(f, f)\right\|_{L^{p}} \leq C_{\mathrm{reg}}\left(\eta, \mathcal{B}_{\mathrm{s}}^{R}\right)\|f\|_{L^{1}}\|f\|_{L^{q}}
$$

Now we need to estimate the remainder $Q_{\mathrm{ns}}^{R,+}=Q^{R,+}-Q_{\mathrm{s}}^{R,+}$. To this aim, we split it as

$$
Q_{\mathrm{ns}}^{R,+}(f, f)=Q_{1}^{R,+}(f, f)+Q_{2}^{R,+}(f, f)+Q_{3}^{R,+}(f, f)+Q_{4}^{R,+}(f, f)
$$

with

$$
\begin{aligned}
Q_{1}^{R,+}(f, f)= & \int_{\mathbb{R}^{d} \times \mathbb{R}^{d}} \mathcal{B}(|y|,|z|) \delta(y \cdot z) \chi^{R}(|z|)\left[\chi^{R}(|y|)-\chi_{\eta}^{R}(|y|)\right] \zeta_{\eta} \Theta_{\eta} f^{\prime} f_{\star}^{\prime} d y d z, \\
Q_{2}^{R,+}(f, f)= & \int_{\mathbb{R}^{d} \times \mathbb{R}^{d}} \mathcal{B}(|y|,|z|) \delta(y \cdot z) \chi_{\eta}^{R}(|y|)\left[\chi^{R}(|z|)-\chi_{\eta}^{R}(|z|)\right] \zeta_{\eta} \Theta_{\eta} f^{\prime} f_{\star}^{\prime} d y d z, \\
Q_{3}^{R,+}(f, f)= & \int_{\mathbb{R}^{d} \times \mathbb{R}^{d}} \mathcal{B}(|y|,|z|) \delta(y \cdot z) \chi_{\eta}^{R}(|z|) \chi_{\eta}^{R}(|y|) \zeta_{\eta} \\
& \times\left[1-\Theta_{\eta}\left(\frac{|y|}{\sqrt{|y|^{2}+|z|^{2}}}\right)\right] f^{\prime} f_{\star}^{\prime} d y d z, \\
Q_{4}^{R,+}(f, f)= & \int_{\mathbb{R}^{d} \times \mathbb{R}^{d}} \mathcal{B}(|y|,|z|) \delta(y \cdot z) \chi_{\eta}^{R}(|z|) \chi_{\eta}^{R}(|y|) \Theta_{\eta}\left(1-\zeta_{\eta}\right) f^{\prime} f_{\star}^{\prime} d y d z .
\end{aligned}
$$

On the one hand, we give a first estimate in $L^{1}\left(\mathcal{D}_{L}\right)$, directly applying the estimate in Lemma 4.1

$$
\left\|Q_{\alpha}^{R,+}\right\|_{L^{1}} \leq C_{1}(R, B)\|f\|_{L^{1}}\|f\|_{L^{1}}, \quad \alpha \in\{1,2,3,4\} .
$$


On the other hand, we treat for instance the operator $Q_{1}^{R,+}(f, f)$ and have for a fixed $v \in \mathcal{D}_{L}$

$$
\begin{aligned}
& \left|Q_{1}^{R,+}(f, f)(v)\right| \\
& \leq \int_{\mathbb{R}^{d} \times \mathbb{R}^{d}} \mathcal{B}(|y|,|z|) \delta(y \cdot z) \chi^{R}(|z|)\left|\chi^{R}(|y|)-\chi_{\eta}^{R}(|y|)\right|\left|f^{\prime}\right|\left|f_{\star}^{\prime}\right| d y d z \\
& \leq \int_{\mathcal{D}_{L}} f^{\prime}\left(\int_{\mathbb{R}^{d}} \mathcal{B}\left(\left|v-v_{\star}^{\prime}\right|,\left|v-v^{\prime}\right|\right) \delta\left(\left(v-v_{\star}^{\prime}\right) \cdot\left(v-v^{\prime}\right)\right)\right. \\
& \left.\quad \times\left|\chi^{R}\left(\left|v-v_{\star}^{\prime}\right|\right)-\chi_{\eta}^{R}\left(\left|v-v_{\star}^{\prime}\right|\right)\right|\left|f_{\star}^{\prime}\right| d v_{\star}^{\prime}\right) d v^{\prime} \\
& \leq\|f\|_{L^{1}}\|f\|_{L^{\infty}} \sup _{y \in \mathbb{R}^{d}}\left(\int_{z \in y^{\perp}} \mathcal{B}^{R}(|z|,|y|)\left|\chi^{R}(|z|)-\chi_{\eta}^{R}(|z|)\right| d z\right) \\
& \leq C_{\infty}(R, B) \eta\|f\|_{L^{1}}\|f\|_{L^{\infty}} .
\end{aligned}
$$

Using similar techniques, we prove that for $\alpha \in\{1,2,3\}$

$$
\left\|Q_{\alpha}^{R,+}\right\|_{L^{\infty}} \leq C_{\infty}(R, B) \eta\|f\|_{L^{1}}\|f\|_{L^{\infty}} .
$$

For the fourth term, we have using the cancellation of the collision kernel $B$ at small relative velocities as $\left|v-v_{*}\right|^{\gamma}$ :

$$
\left\|Q_{4}^{R,+}\right\|_{L^{\infty}} \leq C_{\infty}(R, B) \eta^{\gamma}\|f\|_{L^{1}}\|f\|_{L^{\infty}}
$$

Finally, by the Riesz-Thorin Interpolation Theorem and from (5.7)-(5.8) and (5.6), we deduce that for $p \in[1,+\infty]$ there exist $C_{p}(R, B)>0$ and $\beta \in(0,1]$ such that

$$
\left\|Q_{\alpha}^{R,+}(f, f)\right\|_{L^{p}} \leq C_{p}(R, B) \eta^{\beta}\|f\|_{L^{1}}\|f\|_{L^{p}}
$$

To sum up we have obtained that for all $p>1$ and $\eta>0$ there exist $C>0, q<p$, $\kappa_{0}>0$ and $\beta \in(0,1)$ such that

$$
\left\|Q^{R,+}(f, f)\right\|_{L^{p}} \leq C \eta^{-\kappa_{0}}\|f\|_{L^{1}}\|f\|_{L^{q}}+\eta^{\beta}\|f\|_{L^{1}}\|f\|_{L^{p}} .
$$

The conclusion follows by choosing $\eta$ small enough.

Proof of Proposition 5.1. Now the proof of the propagation of regularity bounds is done exactly as in [20, Section 4 and Subsections 5.1 \& 5.2] (except for the simplification that there are no moment estimates to take care of).

5.2. Entropy - entropy production inequalities. The periodized equation (3.21) preserves non-negativity, and for a non-negative distribution $f$ one can formally compute an $H$ theorem (see [14]):

with

$$
\frac{d}{d t} H(f(t))=-D(f(t)) \leq 0
$$

and

$$
H(f)=\int_{\mathcal{D}_{L}} f \log f d v
$$

$$
\begin{aligned}
D(f) & =-\int_{\mathcal{D}_{L}} Q^{R}(f, f) \log f d v \\
& =\frac{1}{4} \int_{\mathcal{D}_{L} \times \mathcal{C}_{R}}\left(f^{\prime} f_{\star}^{\prime}-f f_{\star}\right) \log \left(\frac{f^{\prime} f_{\star}^{\prime}}{f f_{\star}}\right) \mathcal{B}(y, z) d v d y d z .
\end{aligned}
$$


Then we can state the result which relates the entropy functional $H$ and the entropy production functional $D$ :

Proposition 5.5. We consider the periodized Boltzmann collision operator for some truncation parameter $R>\sqrt{2} L$, and we assume that the collision kernel satisfies $B \geq b_{0}\left|v-v_{\star}\right|^{\gamma}, \gamma>0$, for $\left|v-v_{\star}\right| \leq R$. Then for any $\eta, \alpha>0$ there is $k \in \mathbb{N}$ and $M, K>0$ (depending only on $\eta, \alpha, b_{0}, \gamma, R$ ) such that

$$
D(f) \geq K H\left(f \mid m_{\infty}\right)^{1+\eta}, \quad m_{\infty}=\frac{\rho}{\left|\mathcal{D}_{L}\right|}, \quad \rho=\int_{\mathcal{D}_{L}} f d v,
$$

for any $\alpha \leq f \in L^{1}\left(\mathcal{D}_{L}\right)$ with $H_{\mathrm{per}}^{k}\left(\mathcal{D}_{L}\right)$ norm bounded by $M$.

Remarks. 1. Note that this is a functional inequality independent of the flow of the Boltzmann equation itself.

2. In the case of the classical Boltzmann equation with $v \in \mathbb{R}^{d}$, the entropy production functional controls the relative entropy according to the Maxwellian equilibrium. Here the equilibrium is a constant, defined by the mass of $f$ divided by the total volume of the torus.

We shall adapt the method developed in 32] or later in [34, Proof of Theorem 2.1]. In the first step, we treat the case of a collision kernel $B$ which is uniformly bounded from below. In this case we prove the equivalent of the so-called Cercignani conjecture in the context of the Boltzmann operator periodized in the velocity space.

Remark. Note that the assumption $R \geq \sqrt{2} L$ allows us to replace in the bound from below, when needed, the truncation by the integration over the whole torus.

Lemma 5.6. Let us consider a collision kernel $B$ which satisfies $B \geq b_{0}>0$, $a$ truncation $Q^{R}$ defined by (3.13) or (3.16), together with $R \geq \sqrt{2} L$. Then there is an explicit constant $K$ such that for any $0 \leq f \in L^{1}\left(\mathcal{D}_{L}\right)$ we have

$$
D(f) \geq K H\left(f \mid m_{\infty}\right) \text {. }
$$

Proof. We proceed in several steps.

Step 1. Since the entropy production functional is monotonous in terms of the collision kernel $\mathcal{B}$, it is not a restriction to replace $\mathcal{B}$ by 1 in the sequel for the estimate from below. Moreover it is always possible to bound from below the truncation $\left|v-v^{\prime}\right| \leq R$ and $\left|v-v_{\star}^{\prime}\right| \leq R$ (in case we performed the truncation for the fast spectral method) by the classical truncation $\left|v-v_{\star}\right| \leq R$.

Step 2. Using Jensen's inequality on the sphere integration (coming back to the classical truncation by the previous remark) and the joint convexity of the function $(X, Y) \mapsto(X-Y)(\log X-\log Y)$ on $\mathbb{R}_{+} \times \mathbb{R}_{+}$, we compute

$$
D(f) \geq C \int_{\mathcal{D}_{L}} \int_{\mathcal{B}(v, R)}(F-G) \log \frac{F}{G} d v_{\star} d v=: \bar{D}(f),
$$

where $F=f f_{\star}$ and

$$
G=\frac{1}{\left|\mathbb{S}^{d-1}\right|} \int_{\mathbb{S}^{d-1}} f^{\prime} f_{\star}^{\prime} d \sigma .
$$

Let us study more precisely the function $G$. As it was already observed by Boltzmann himself, the function $G$ only depends on $v+v_{\star}$ and $\left(|v|^{2}+\left|v_{\star}\right|^{2}\right) / 2$. Moreover, here it is also periodic on the torus $\mathcal{D}_{L}$ since $f$ is periodic. It implies (when $f$ is 
smooth, but we can always use mollifications to relax this assumption here) that it in fact only depends on $v+v_{\star}$.

Step 3. Let us denote by $S_{t}$ the semi-group of the heat equation on $L^{1}\left(\mathcal{D}_{L}\right)$ (and for brevity we keep the same notation for its semi-group in $L^{1}\left(\mathcal{D}_{L}^{2}\right)$ ). Then the semi-group is compatible with the symmetries in the sense that

$$
S_{t}\left(f f_{\star}\right)=S_{t}(f) S_{t}\left(f_{\star}\right)
$$

and $S_{t} G$ only depends on $v+v_{\star}$ (this follows from a straightforward computation using the explicit formula for the Green kernel of $S_{t}$ ).

Step 4. Then we have the following computation as in [34:

$$
\left.\frac{d}{d t}\right|_{t=0}\left[S_{t}\left((F-G) \log \frac{F}{G}\right)-\left(S_{t} F-S_{t} G\right) \log \frac{S_{t} F}{S_{t} G}\right]=\left|\frac{\nabla F}{F}-\frac{\nabla G}{G}\right|^{2}(F+G),
$$

where $\nabla$ denotes the gradient with respect to $\left(v, v_{\star}\right) \in \mathbb{R}^{2 d}$.

Then we bound from below the truncation $\mathbf{1}_{B(v, R)}\left(v_{\star}\right)$ by the integration over the whole torus for $v_{\star}$ (since $R$ is large enough), and we compute

$$
\begin{aligned}
-\left.\frac{d}{d t}\right|_{t=0} \bar{D}\left(S_{t} f\right)= & \int_{\mathcal{D}_{L} \times \mathcal{D}_{L}}\left|\frac{\nabla F}{F}-\frac{\nabla G}{G}\right|^{2}(F+G) d v d v_{\star} \\
& -\int_{\mathcal{D}_{L} \times \mathcal{D}_{L}} \Delta\left((F-G) \log \frac{F}{G}\right) d v d v_{\star} \\
= & \int_{\mathcal{D}_{L} \times \mathcal{D}_{L}}\left|\frac{\nabla F}{F}-\frac{\nabla G}{G}\right|^{2}(F+G) d v d v_{\star} .
\end{aligned}
$$

We deduce by the semi-group property that for all $t>0$

$$
-\frac{d}{d t} \bar{D}\left(S_{t} f\right) \geq \int_{\mathcal{D}_{L} \times \mathcal{D}_{L}}\left|\frac{\nabla S_{t} F}{S_{t} F}-\frac{\nabla S_{t} G}{S_{t} G}\right|^{2}\left(S_{t} F+S_{t} G\right) d v d v_{\star}
$$

and therefore

$$
\bar{D}(f) \geq \int_{0}^{+\infty}\left(\int_{\mathcal{D}_{L} \times \mathcal{D}_{L}}\left|\frac{\nabla S_{t} F}{S_{t} F}-\frac{\nabla S_{t} G}{S_{t} G}\right|^{2}\left(S_{t} F+S_{t} G\right) d v d v_{\star}\right) d t .
$$

Step 5 . We now use the fact that the operator

$$
P:\left\{\begin{array}{lll}
\mathbb{R}^{d} \times \mathbb{R}^{d} & \mapsto \mathbb{R}^{d} \\
(A, B) & \mapsto(A-B)
\end{array}\right.
$$

is bounded from $\mathcal{D}_{L} \times \mathcal{D}_{L}$ to $\mathcal{D}_{L}$. Hence

$$
\left|\frac{\nabla S_{t} F}{S_{t} F}-\frac{\nabla S_{t} G}{S_{t} G}\right|^{2} \geq C_{1}\left|\frac{P \nabla S_{t} F}{S_{t} F}\right|^{2}
$$

since $P \nabla S_{t} G=\nabla_{v} G-\nabla_{v_{\star}} G=0$ from the fact that $G$ only depends on $v+v_{\star}$. We deduce that

$$
\bar{D}(f) \geq C \int_{0}^{+\infty}\left(\int_{\mathcal{D}_{L} \times \mathcal{D}_{L}}\left|\frac{\nabla_{v} S_{t} f}{S_{t} f}-\left(\frac{\nabla_{v_{\star}} S_{t} f}{S_{t} f}\right)_{\star}\right|^{2}\left(S_{t} f S_{t} f_{\star}+S_{t} G\right) d v d v_{\star}\right) d t
$$


and thus (dropping the term $S_{t} G$ )

$$
\bar{D}(f) \geq C \int_{0}^{+\infty}\left(\int_{\mathcal{D}_{L} \times \mathcal{D}_{L}}\left|\frac{\nabla_{v} S_{t} f}{S_{t} f}-\left(\frac{\nabla_{v_{\star}} S_{t} f}{S_{t} f}\right)_{\star}\right|^{2} S_{t} f S_{t} f_{\star} d v d v_{\star}\right) d t .
$$

Step 6. From now on the proof departs slightly more from [34]: it is simpler since we are in the torus and we have more symmetries. Let us show the following functional inequality: for any smooth non-negative function $h$,

$$
\int_{\mathcal{D}_{L} \times \mathcal{D}_{L}}\left|\frac{\nabla_{v} h}{h}-\left(\frac{\nabla_{v_{\star}} h}{h}\right)_{\star}\right|^{2} h h_{\star} d v d v_{\star} \geq C_{2} I\left(h \mid m_{\infty}\right)
$$

where

$$
I(h \mid g)=\int_{\mathcal{D}_{L}} h\left|\nabla_{v} \log \frac{h}{g}\right|^{2} d v
$$

The proof only amounts to Jensen's inequality on the variable $v_{\star}$ : since $\int_{\mathcal{D}_{L}} h d v=$ $\rho$,

$$
\begin{aligned}
\int_{\mathcal{D}_{L} \times \mathcal{D}_{L}} & \left|\frac{\nabla_{v} h}{h}-\left(\frac{\nabla_{v_{\star}} h}{h}\right)_{\star}\right|^{2} h h_{\star} d v d v_{\star} \\
& \geq \frac{C}{\rho} \int_{\mathcal{D}_{L}}\left|\int_{\in \mathcal{D}_{L}}\left(\frac{\nabla_{v} h}{h}-\left(\frac{\nabla_{v_{\star}} h}{h}\right)_{\star}\right) h_{\star} d v_{\star}\right|^{2} h d v .
\end{aligned}
$$

Then as

$$
\int_{\mathcal{D}_{L}} \nabla h_{\star} d v_{\star}=0
$$

we deduce that

$$
\int_{\mathcal{D}_{L} \times \mathcal{D}_{L}}\left|\frac{\nabla_{v} h}{h}-\left(\frac{\nabla_{v_{\star}} h}{h}\right)_{\star}\right|^{2} h h_{\star} \geq C \int_{\mathcal{D}_{L}}\left|\frac{\nabla_{v} h}{h}\right|^{2} h d v=C I\left(h \mid m_{\infty}\right) .
$$

Step 7 . So far we have proved

$$
D(f) \geq \bar{D}(f) \geq C_{3} \int_{0}^{+\infty} I\left(S_{t} f \mid m_{\infty}\right) d t .
$$

Then a trivial computation shows that

$$
\frac{d}{d t} H\left(S_{t} f \mid m_{\infty}\right)=-I\left(S_{t} f \mid m_{\infty}\right) .
$$

Moreover, from the explicit formula for $S_{t}$ we have

$$
H\left(S_{t} f \mid m_{\infty}\right) \stackrel{t \rightarrow+\infty}{\longrightarrow} 0,
$$

and thus we finally obtain

$$
D(f) \geq \bar{D}(f) \geq C_{3}\left(H\left(S_{0} f \mid m_{\infty}\right)-0\right) \geq C_{3} H\left(f \mid m_{\infty}\right) .
$$

Now we are ready to prove Proposition 5.5. Since we deal with a bounded velocity domain we do not care about possible decay of the collision kernel at large relative velocity (as for soft potentials), and the only cancellation we have to treat is the one for zero relative velocities. 
Proof of Proposition 5.5. We only mention the difference as compared to the previous proof.

The reduction to a collision kernel uniformly bounded from below studied in Lemma 5.6 is done as in [34, Theorem 4.1]: write for some small $\delta>0$

$$
B\left(\left|v-v_{\star}\right|\right) \geq \delta^{\gamma}\left(B_{0}-\mathbf{1}_{\mathcal{B}(0, \delta)}\left(\left|v-v_{\star}\right|\right)\right),
$$

where $B_{0} \geq b_{0}>0$, and deduce that

$$
D(f) \geq \delta^{\gamma}\left(D_{0}(f)-\tilde{D}_{\delta}(f)\right),
$$

where $D_{0}$ is the entropy production functional corresponding to $B_{0}$ and

$$
\tilde{D}_{\delta}=\frac{1}{4} \int_{\mathcal{D}_{L} \times \mathcal{D}_{L}} \int_{\mathbb{S}^{d-1}}\left(f^{\prime} f_{\star}^{\prime}-f f_{\star}\right) \log \frac{f^{\prime} f_{\star}^{\prime}}{f f_{\star}} \mathbf{1}_{\mathcal{B}(0, \delta)}\left(\left|v-v_{\star}\right|\right) d \sigma d v d v_{\star} .
$$

Then we have the following lemma, which is proved exactly as [34, Theorem 4.2]. It is even simpler since Maxwellians are replaced by constant functions and the study of the tail is not needed (we omit the proof for brevity).

Lemma 5.7. For any $\varepsilon \in(0,1)$ and $\alpha>0$, there are constants of smoothness $k, M$ and some corresponding constant $C_{\mathrm{diag}}>0$ such that

$$
\tilde{D}_{\delta} \leq C_{\text {diag }} H\left(f \mid m_{\infty}\right)^{1-\varepsilon} \delta^{d / 4}
$$

for any $\alpha \leq f \in L^{1}\left(\mathcal{D}_{L}\right)$ with an $H_{\mathrm{per}}^{k}\left(\mathcal{D}_{L}\right)$ norm bounded by $M$.

But since we have

$$
D_{0}(f) \geq C H\left(f \mid m_{\infty}\right)
$$

from Lemma [5.6] it is straightforward to obtain the result by correctly choosing the parameter $\delta$.

Now we can proceed to the proof of Theorem 3.1

5.3. Proof of the global in time stability. In this subsection we shall turn to the question of obtaining uniform bounds as well as global existence in order to conclude the proof of Theorem 3.1. Indeed, in Section 4 the smallness assumption on the truncation parameter $\varepsilon$ a priori depends on $T$ and could go to 0 as $T$ goes to infinity, since it depends on regularity bounds growing exponentially in time.

In order to overcome this difficulty, we shall combine the following arguments:

- for the unperturbed problem (3.21) we have a Liapunov structure, and the solution converges to a unique prescribed equilibrium from the regularity and entropy production studies;

- the equilibrium distribution of the unperturbed problem (3.21) (that is, the constant functions on the torus) is also the equilibrium distribution of the perturbed problem (3.17);

- by taking the size of the perturbation small enough (measured in terms of $\varepsilon$ ), it is possible to construct a solution to the perturbed problem on an arbitrarily large time interval $[0, T]$, on which moreover the perturbed solution remains close to the unperturbed solution (3.21), say in Sobolev norms;

- finally the constant equilibrium functions are non-linearly stable for the perturbed problem, with a stability domain independent of the size of the perturbation. 
Hence we shall deduce that as soon as the time for which the perturbed solution departs from the unperturbed solution is larger that the time-scale of relaxation to equilibrium for the unperturbed problem (3.21), the perturbed solution shall be trapped by the stability domain of the equilibrium before instability due to the perturbation can develop. Let us formalize these arguments in a proof:

Proof of the global stability and asymptotic behavior in Theorem 3.1. Let us consider some initial datum $0 \leq f_{0} \in L^{1}$ which belongs to $H_{\mathrm{per}}^{k}\left(\mathcal{D}_{L}\right)$ and some smooth balanced perturbations $f_{0, \varepsilon}$ of it. These perturbations have the same mass and therefore are corresponding to the same equilibrium (this is the reason for this assumption).

Since the smooth balanced perturbation preserves the constant equilibrium of (3.21) and is non-linearly locally stable in any $H_{\text {per }}^{k}\left(\mathcal{D}_{L}\right)$, we fix a $\eta>0$ such that the constant distribution $m_{\infty}=\rho /\left|\mathcal{D}_{L}\right|$ associated to the mass $\rho$ of $f_{0}$ on the torus has attraction domain with size $\eta$ in $H_{\text {per }}^{k}\left(\mathcal{D}_{L}\right)$ for the perturbed problem (3.17).

On the one hand, we show that there exists a unique solution $f(t)$ to (3.21), and from Proposition 5.1 we obtain uniform regularity bounds for all $t \geq 0$ :

$$
\|f(t)\|_{H_{\mathrm{per}}^{k}} \leq C
$$

Moreover, from Proposition 5.5 there exists a time $T_{0}$ such that the solution $f(t)$ is $\eta / 2$-close to the equilibrium in $H_{\text {per }}^{p}\left(\mathcal{D}_{L}\right)(p<k)$ for $t \geq T_{0}$ (using the CsiszárKullback inequality in the torus; see [5, Theorem 1] for instance):

$$
\left\|f(t)-m_{\infty}\right\|_{H_{\mathrm{per}}^{p}} \leq \eta / 2 .
$$

On the other hand, applying Proposition 4.6 with $T=T_{0}$, we prove that there exists $\hat{\varepsilon}$, which only depends on the $H_{\text {per }}^{k}\left(\mathcal{D}_{L}\right)$ and $L^{1}\left(\mathcal{D}_{L}\right)$ norms of $f_{0}$, such that for all $\varepsilon$ such that $0<\varepsilon<\hat{\varepsilon}$, there exists a unique smooth solution $f_{\varepsilon}(t) \in H_{\text {per }}^{k}\left(\mathcal{D}_{L}\right)$ to 3.17 on $\left[0, T_{0}\right]$ such that

$$
\forall t \in\left[0, T_{0}\right], \quad\left\|f_{\varepsilon}(t)\right\|_{H_{\mathrm{per}}^{k}} \leq C\left(T_{0}\right)
$$

and (for any $p<k$ )

$$
\left\|f(t)-f_{\varepsilon}(t)\right\|_{H_{\mathrm{per}}^{p}} \leq \bar{\varphi}_{T_{0}}(\varepsilon),
$$

where $f(t)$ is a solution to (3.21) and $\bar{\varphi}_{T_{0}}(\varepsilon)$ goes to zero when $\varepsilon$ goes to zero.

Then, we fix a perturbation parameter $\hat{\varepsilon}$ small enough such that for $\varepsilon \in(0, \hat{\varepsilon})$ the perturbed solution $f_{\varepsilon}$ satisfies

$$
\left\|f(t)-f_{\varepsilon}(t)\right\|_{H_{\mathrm{per}}^{p}} \leq \eta / 2 .
$$

Finally, at time $T_{0}$ the perturbed solution $f_{\varepsilon}$ belongs to the stability domain of the constant distribution for the perturbed problem and it is trapped.

Therefore there exists a unique global smooth solution $f_{\varepsilon}$, which is uniformly bounded for all $t \geq 0$ and such that for any $p<k$

$$
\left\|f_{\varepsilon}(t)\right\|_{H_{\mathrm{per}}^{p}} \leq \max \left(C\left(T_{0}\right), C+\eta\right) .
$$

This achieves the proof of $(i),(i i),(i i i)$ and $(i v)$. 


\section{Applichtion: Stability And Convergence of SPeCtral methods}

In this section we consider the following spectral approximation of (3.21):

$$
\frac{\partial f_{N}}{\partial t}=\mathcal{P}_{N} Q^{R}\left(f_{N}, f_{N}\right)
$$

where $\mathcal{P}_{N}$ denotes the orthogonal projection on $\mathbb{P}_{N}$ in $L^{2}\left(\mathcal{D}_{L}\right)$ (the space of trigonometric polynomials with degree less than at most $N$ in each direction).

The goal of this section is to prove the following theorem:

Theorem 6.1. Consider any non-negative initial datum $f_{0} \in H_{\mathrm{per}}^{k}\left(\mathcal{D}_{L}\right)$, with $k>$ $d / 2$, which is not zero everywhere. Then there exists $N_{0} \in \mathbb{N}$ (depending on the mass and $H_{\text {per }}^{k}\left(\mathcal{D}_{L}\right)$ norm of $f$ ) such that for all $N \geq N_{0}$ :

(i) there is a unique global solution $f_{N}=f_{N}(t, \cdot)$ to the following problem:

$$
\left\{\begin{array}{l}
\frac{\partial f_{N}}{\partial t}=\mathcal{P}_{N} Q^{R}\left(f_{N}, f_{N}\right), \\
f_{N}(t=0)=\mathcal{P}_{N} f_{0}
\end{array}\right.
$$

(ii) for any $p<k$, there exists $C>0$ such that

$$
\forall t \geq 0, \quad\left\|f_{N}(t, \cdot)\right\|_{H_{\mathrm{per}}^{k}} \leq C
$$

(iii) this solution is everywhere positive for time large enough, and the mass of its negative values can be made uniformly (in times) $L^{\infty}$ small as $N \rightarrow \infty$;

(iv) this solution $f_{N}$ converges to $f(t)$ the solution to (3.21) with the spectral accuracy, uniformly in time;

$(v)$ this solution converges exponentially fast to a constant solution on the torus prescribed by the mass conservation law.

To prove Theorem 6.1, we want to apply Theorem 3.1 with the perturbation

$$
P_{N}^{R}\left(f_{N}\right):=\mathcal{P}_{N} Q^{R}\left(f_{N}, f_{N}\right)-Q^{R}\left(f_{N}, f_{N}\right),
$$

which preserves the mass:

$$
\int_{\mathcal{D}_{L}} P_{N}^{R}\left(f_{N}\right) d v=\int_{\mathcal{D}_{L}}\left(\mathcal{P}_{N} Q^{R}\left(f_{N}, f_{N}\right)-Q^{R}\left(f_{N}, f_{N}\right)\right) d v=0 .
$$

In the next lemma, we prove a consistency and smoothness result for this approximation.

Lemma 6.2. Consider a non-negative function $f \in H_{\mathrm{per}}^{k}\left(\mathcal{D}_{L}\right)$, with $k>d / 2$, which is not zero everywhere. Then there exists $C>0$ depending only on the collision kernel $B$ and the truncation such that for all $p \in[0, k]$ we have

$$
\left\|P_{N}^{R}(f)\right\|_{H_{\mathrm{per}}^{p}} \leq C\|f\|_{L^{1}}\|f\|_{H_{\mathrm{per}}^{p}} .
$$

Moreover, for all $p \in[0, k]$,

$$
\left\|P_{N}^{R}(f)\right\|_{H_{\mathrm{per}}^{p}} \leq C\|f\|_{L^{1}} \frac{\|f\|_{H_{\mathrm{per}}^{k}}}{N^{k-p}} .
$$


Proof. First, we split the operator $P_{N}$ as

$$
\left\|P_{N}^{R}(f)\right\|_{H_{\mathrm{per}}^{p}} \leq\left\|Q^{R}(f, f)\right\|_{H_{\mathrm{per}}^{p}}+\left\|\mathcal{P}_{N} Q^{R}(f, f)\right\|_{H_{\mathrm{per}}^{p}} .
$$

As in the proof of Lemma 4.1 we get that for all $p \in[0, k]$

$$
\left\|Q^{R}(f, f)\right\|_{H_{\mathrm{per}}^{p}}^{2} \leq C_{p}(R, B)\|f\|_{L^{1}}^{2}\|f\|_{H_{\mathrm{per}}^{p}}^{2} .
$$

Concerning the interpolation error estimate, the following result holds. If $u \in$ $H_{\mathrm{per}}^{p}\left(\mathcal{D}_{L}\right)$ for some $p \geq 1$, then

$$
\left\|u-\mathcal{P}_{N} u\right\|_{H_{\mathrm{per}}^{p}} \leq \frac{C}{N^{k-p}}\|u\|_{H_{\mathrm{per}}^{k}} .
$$

Then, taking $p=k$ in the latter inequality and from (6.4) we obtain

$$
\left\|\mathcal{P}_{N} Q^{R}(f, f)\right\|_{H_{\mathrm{per}}^{p}}^{2} \leq\left\|Q^{R}(f, f)\right\|_{H_{\mathrm{per}}^{p}}^{2} \leq C_{p}(R, B)\|f\|_{L^{1}}^{2}\|f\|_{H_{\mathrm{per}}^{p}}^{2} .
$$

Gathering (6.4) and (6.6), we finally get

$$
\left\|P_{N}^{R}(f)\right\|_{H_{\mathrm{per}}^{p}} \leq C_{p}(R, B)\|f\|_{L^{1}}\|f\|_{H_{\mathrm{per}}^{p}} .
$$

Moreover, again using the error estimate (6.5), it yields

$$
\begin{aligned}
\left\|P_{N}^{R}(f)\right\|_{H_{\mathrm{per}}^{p}} & \leq C \frac{\left\|Q^{R}(f, f)\right\|_{H_{\mathrm{per}}^{k}}}{N^{k-p}} \\
& \leq C(R, B)\|f\|_{L^{1}} \frac{\|f\|_{H_{\mathrm{per}}^{k}}}{N^{k-p}} .
\end{aligned}
$$

Let us now perform a linearized study of the perturbed equation (6.1) by classical Fourier-basis decomposition. The only equilibrium distributions of the equation (3.21) are the constant, prescribed by the mass conservation. Let us consider the linearized version of the perturbed equation (6.1) around such a constant $m_{\infty}$ :

$$
\frac{\partial f}{\partial t}=m_{\infty} \mathcal{L}^{N, R}(f)
$$

where

$$
\mathcal{L}^{N, R}(f)=\mathcal{P}_{N}\left[Q^{R}(f, 1)+Q^{R}(1, f)\right] .
$$

Let us prove the following lemma:

Lemma 6.3. The operator $\mathcal{L}^{N, R}$ is bounded and self-adjoint in $L^{2}\left(\mathcal{D}_{L}\right)$. Moreover, it is non-negative, its null space is given by the constant functions, and it has a spectral gap $\lambda>0$. As a consequence, the constants are nonlinearly locally stable in any $H_{\mathrm{per}}^{k}\left(\mathcal{D}_{L}\right)$ for the equation (6.1), with a stability domain independent on $N$.

Proof. The boundedness is trivial. Then, the periodized operator $Q^{R}$ is translation invariant, which implies that the Fourier modes

$$
e_{k}(v)=\frac{\exp \left[i \frac{\pi}{L}(k \cdot v)\right]}{\left|\mathcal{D}_{L}\right|}
$$

for $k \in \mathbb{Z}^{d}$ are trivially eigenfunctions of $\mathcal{L}^{N, R}$. This provides a complete orthonormal eigenbasis in $L^{2}\left(\mathcal{D}_{L}\right)$. A trivial computation yields

$\mathcal{L}^{N, R}\left(e_{k}\right)=a_{k} e_{k} \mathbf{1}_{|k| \leq N}$ with $a_{k}:=-\int_{\mathcal{C}_{R}}\left[1+e_{k}(y+z)-e_{k}(y)-e_{k}(z)\right] \mathcal{B}(y, z) d y d z$. 
In particular we deduce that $\mathcal{L}^{N, R}=\mathcal{L}^{N, R} \mathcal{P}_{N}$, and the self-adjointness comes from the following identity obtained by the usual changes of variables:

$$
\begin{aligned}
\int_{\mathcal{D}_{L}} \mathcal{L}^{N, R}(f) g d v=-\frac{1}{4} \int_{\mathcal{D}_{L}} & \mathcal{C}_{R}\left[\mathcal{P}_{N} f^{\prime}+\mathcal{P}_{N} f_{\star}^{\prime}-\mathcal{P}_{N} f-\mathcal{P}_{N} f_{\star}\right] \\
& \times\left[\mathcal{P}_{N} g^{\prime}+\mathcal{P}_{N} g_{\star}^{\prime}-\mathcal{P}_{N} g-\mathcal{P}_{N} g_{\star}\right] \mathcal{B}(y, z) d y d z d v .
\end{aligned}
$$

Another formula for $a_{k}$ is readily deduced from the previous representation:

$$
a_{k}=-\frac{1}{4} \int_{\mathcal{D}_{L} \times \mathcal{C}_{R}}\left|\left(e_{k}\right)^{\prime}+\left(e_{k}\right)_{\star}^{\prime}-\left(e_{k}\right)-\left(e_{k}\right)_{\star}\right|^{2} \mathcal{B}(y, z) d y d z d v .
$$

One sees from the second representation that $a_{k}=a_{-k} \leq 0$ for any $k \in \mathbb{Z}^{d}$, and from the first representation and Lebesgue theorem it is easily seen that for $|k| \rightarrow \infty$ the coefficients $a_{k}$ converge as

$$
a_{k} \underset{|k| \rightarrow \infty}{\longrightarrow} a_{\infty}=-\int_{\mathcal{C}_{R}} \mathcal{B}(y, z) d y d z \in(-\infty, 0) .
$$

Hence we deduce that $a_{k} \in\left[a_{\infty}, 0\right]$ for any $k \in \mathbb{Z}^{d}$, with asymptotic convergence towards $a_{\infty}$ for large $k$. Moreover, the null space can be computed: for some smooth periodic function $\phi$, the equation

$$
\phi^{\prime}+\phi_{\star}^{\prime}-\phi-\phi_{\star}=0
$$

implies that the third-order derivative of $\phi$ is zero, and the periodicity then imposes that it is a constant. Thus the null space is spanned by $e_{0}$. It concludes the proof of the existence of a spectral gap

$$
\lambda_{N}:=\min \left\{\left|a_{k}\right|, k \in[|-N, N|]^{d}, k \neq 0\right\}>0,
$$

which is uniformly bounded from below as $N \rightarrow+\infty$, since

$$
\lambda_{N} \rightarrow \lambda_{\infty}:=\min \left\{\left|a_{k}\right|, k \in \mathbb{Z}^{d}, k \neq 0\right\}>0 .
$$

The non-linear stability in $L^{2}$ comes from the fact that for the perturbation $h=f-m_{\infty}$, we have the following control on the bilinear part:

$$
\left\|\mathcal{P}_{N} Q^{R}(h, h)\right\|_{H_{\mathrm{per}}^{k}} \leq C_{\mathcal{B}, R}\|h\|_{L^{2}}^{2}
$$

for some given constant $C_{\mathcal{B}, R}>0$ independent on $N$.

Finally using the eigenbasis of the Fourier modes, a similar study can be performed in any Sobolev space $H_{\mathrm{per}}^{k}\left(\mathcal{D}_{L}\right)$.

Remark. Exact computations could be made for particular physical collision kernels $\mathcal{B}$ (in a similar way as the computation of the kernel modes $\beta(l, m)$ in $[11,12,21$, 14]).

6.1. Proof of Theorem 6.1. Consider the numerical solution $f_{N}$ given by solving (6.1). We can formulate the problem as a perturbation of the truncated Boltzmann equation. Indeed, setting

$$
P_{N}\left(f_{N}\right)=-\left(I d-\mathcal{P}_{N}\right) Q^{R}\left(f_{N}, f_{N}\right),
$$

the problem (6.1) can be written as

$$
\frac{\partial f_{N}}{\partial t}=Q^{R}\left(f_{N}, f_{N}\right)+P_{N}\left(f_{N}\right) .
$$


Then, applying Lemma 6.2 the perturbation $P_{N}$ satisfies the assumptions of Theorem 3.1. Moreover, since $f_{0} \in H_{\mathrm{per}}^{k}\left(\mathcal{D}_{L}\right)$, we have straightforwardly

$$
\left\|f_{N}(0)\right\|_{H_{\mathrm{per}}^{p}} \leq\left\|f_{0}\right\|_{H_{\mathrm{per}}^{p}}, \quad\left\|f_{N}(0)-f_{0}\right\|_{H_{\mathrm{per}}^{p}} \rightarrow 0 .
$$

Hence, we can directly apply Theorem 3.1 to the perturbation $P_{N}$, which proves that there exists $N_{0}$ large enough and only depending on $f_{0}$, the kernel $B$ and the truncation, such that for all $N \geq N_{0}$, the perturbed system admits a unique uniformly smooth solution, which converges to a constant and satisfies all the points in Theorem 3.1.

\section{REFERENCES}

[1] Bird, G. A.: Molecular gas dynamics. Clarendon Press, Oxford (1994).

[2] Bobylev, A. V.: The theory of the nonlinear spatially uniform Boltzmann equation for Maxwell molecules. Math. Phys. Reviews, vol. 7 (1988) pp. 111-233. MR1128328(92m:82112)

[3] Bobylev, A. V. and Rjasanow, S.: Difference scheme for the Boltzmann equation based on the fast Fourier transform. European J. Mech. B Fluids 16 (1997) pp. 293-306. MR 1439069 (98c:82057)

[4] Bobylev, A. V. and Rjasanow, S.: Fast deterministic method of solving the Boltzmann equation for hard spheres. Eur. J. Mech. B Fluids 18 (1999) pp. 869-887. MR1728639 (2001c:76109)

[5] Bolley, F., Villani, C.: Weighted Csiszár-Kullback-Pinsker inequalities and applications to transportation inequalities. Ann. Fac. Sci. Toulouse Math. (6) 14 (2005) pp. 331-352. MR2172583 (2006j:60018)

[6] Canuto, C., Hussaini, M. Y., Quarteroni, A. and Zang, T. A.: Spectral methods in fluid dynamics. Springer Series in Computational Physics, Springer-Verlag, New York 1988). MR917480 (89m:76004)

[7] Carleman T.: Sur la théorie de l'équation intégrodifférentielle de Boltzmann. Acta Math. 60 (1932).

[8] Cercignani, C., Illner, R. and Pulvirenti, M.: The Mathematical Theory of Dilute Gases. Appl. Math. Sci. 106, Springer-Verlag, New York (1994). MR1307620 (96g:82046)

[9] Fainsilber, L., Kurlberg, P. and Wennberg, B.; Lattice points on circles and discrete velocity models for the Boltzmann equation. SIAM J. Math. Anal. 37 (2006) pp. 1903-1922. MR2213399 (2007f:11116)

[10] Filbet, F. and Pareschi, L.: A numerical method for the accurate solution of the FokkerPlanck-Landau equation in the non homogeneous case. J. Comput. Phys. 179 (2002) pp. 1-26. MR.1906573(2003c:82085)

[11] Filbet, F. and Russo, G.: High order numerical methods for the space non-homogeneous Boltzmann equation. J. Comput. Phys. 186 (2003) pp. 457-480. MR1973198(2004c:82116)

[12] Filbet, F. and Russo, G.: Accurate numerical methods for the Boltzmann equation. Modeling and computational methods for kinetic equations, pp. 117-145, Model. Simul. Sci. Eng. Technol., Birkhäuser Boston, Boston, MA 2004. MR2068582 (2005d:82117)

[13] Filbet, F., Pareschi, L. and Toscani, G.: Accurate numerical methods for the collisional motion of (heated) granular flows. J. Comput. Phys. 202 (2005) pp. 216-235. MR2102883 (2005g:82122)

[14] Filbet, F., Mouhot, C. and Pareschi, L.: Solving the Boltzmann equation in $N \log _{2}$ N. SIAM J. Sci. Comput. 28 (2006) pp. 1029-1053 MR2240802(2007c:82076)

[15] Ibragimov, I. and Rjasanow, S.: Numerical solution of the Boltzmann equation on the uniform grid. Computing 69 (2002) pp. 163-186. MR.1954793 (2004i:82060)

[16] Lions, P.-L.: Compactness in Boltzmann's equation via Fourier integral operators and applications, I. J. Math. Kyoto Univ. 34 (1994) pp. 391-427. MR.1284432 (96f:35133)

[17] Lions, P.-L.: Compactness in Boltzmann's equation via Fourier integral operators and applications, II. J. Math. Kyoto Univ. 34 (1994) pp. 429-461. MR1284432 (96f:35133)

[18] Mischler, S. and Mouhot, C.: Cooling process for inelastic Boltzmann equations for hard spheres. II. Self-similar solutions and tail behavior. J. Stat. Phys. 124 (2006) pp. 703-746. MR2264623 (2007g:82054) 
[19] Mouhot, C.: Quantitative lower bounds for the full Boltzmann equation. I. Periodic boundary conditions. Comm. Partial Differential Equations 30 (2005) pp. 881-917. MR2153518 (2006a:76096)

[20] Mouhot, C. and Villani, C.: Regularity theory for the spatially homogeneous Boltzmann equation with cut-off. Arch. Rational Mech. Anal. 173 (2004) pp. 169-212. MR 2081030 (2006f:82073)

[21] Mouhot, C. and Pareschi, L.: Fast algorithms for computing the Boltzmann collision operator. Math. Comp. 75 (2006) pp. 1833-1852. MR2240637 (2007d:65095)

[22] Nanbu, K.: Direct simulation scheme derived from the Boltzmann equation. I. Monocomponent Gases. J. Phys. Soc. Japan 52 (1983) pp. 2042-2049.

[23] Palczewski, A., Schneider, J. and Bobylev, A. V.: A consistency result for a discretevelocity model of the Boltzmann equation. SIAM J. Numer. Anal. 34 (1997) pp. 1865-1883. MR 1472201 (99d:82068)

[24] Palczewski, A. and Schneider, J.: Existence, stability, and convergence of solutions of discrete velocity models to the Boltzmann equation. J. Statist. Phys. 91 (1998) pp. 307-326. MR:1632506 (99g:82072)

[25] Panferov, V. A. and Heintz, A. G.: A new consistent discrete-velocity model for the Boltzmann equation. Math. Methods Appl. Sci. 25 (2002) pp. 571-593. MR.1895119(2003d:82100)

[26] Pareschi, L. and Perthame, B.: A Fourier spectral method for homogeneous Boltzmann equations. Trans. Theo. Stat. Phys. 25 (1996) pp. 369-382. MR.1407541 (97j:82133)

[27] Pareschi, L. and Russo, G.: Numerical solution of the Boltzmann equation I. Spectrally accurate approximation of the collision operator. SIAM J. Numer. Anal. 37 (2000) pp. 12171245. MR1756425 (2001g:65175)

[28] Pareschi, L., Russo, G. and Toscani, G.: Fast spectral methods for the Fokker-Planck-Landau collision operator. J. Comput. Phys. 165 (2000) pp. 216-236. MR:1795398(2001i:65112)

[29] Pareschi, L. and Russo, G.: On the stability of spectral methods for the homogeneous Boltzmann equation. Trans. Theo. Stat. Phys. 29 (2000) pp. 431-447. MR.1770438(2001c:82070)

[30] Pareschi, L., Toscani, G. and Villani, C.: Spectral methods for the non cut-off Boltzmann equation and numerical grazing collision limit, Numer. Math. 93 (2003) pp. 527-548. MR 1953752 (2003k:65128)

[31] Pulvirenti, A. and Wennberg, B.: A Maxwellian lower bound for solutions to the Boltzmann equation. Comm. Math. Phys. 183 (1997) pp. 145-160. MR1461954(99f:82056)

[32] Toscani, G. and Villani, C.: Sharp entropy dissipation bounds and explicit rate of trend to equilibrium for the spatially homogeneous Boltzmann equation, Commun. Math. Phys. 203 (1999) pp. 667-706. MR1700142(2000e:82039)

[33] Villani, C.: A survey of mathematical topics in kinetic theory. Handbook of fluid mechanics, S. Friedlander and D. Serre, Eds. Elsevier Publ. (2002).

[34] Villani, C.: Cercignani's conjecture is sometimes true and always almost true. Comm. Math. Phys. 234 (2003) pp. 455-490. MR1964379 (2004b:82048)

[35] Wennberg, B.; Regularity in the Boltzmann equation and the Radon transform. Comm. Partial Differential Equations 19 (1994), pp. 2057-2074. MR1301182 (95k:76103)

[36] Wennberg, B.: The geometry of binary collisions and generalized Radon transforms. Arch. Rational Mech. Anal. 139 (1997) pp. 291-302. MR1480243 (98k:82166)

Université de Lyon, Institut Camille Jordan, CNRS UMR 5208, Université de Lyon 1, INSAL, ECL, 43 Boulevard 11 novembre 1918, F-69622 Villeurbanne Cedex, France E-mail address: filbet@math.univ-lyon1.fr

Centre for Mathematical Sciences, University of Cambridge, DAMTP, Wilberforce Road, Cambridge CB3 0WA, England

Current address: CNRS \& École Normale Supérieure, DMA, UMR CNRS 8553, 45, rue d'Ulm, F-75230 Paris cedex 05, FRANCE

E-mail address: Clement.Mouhot@ens.fr 